Supporting Information for J. Org. Chem.

\title{
A Facile One-Pot Preparation of Alkyl Aminoaryl Sulfides for the Synthesis of GW7647 as an Agonist of Peroxisome Proliferator-Activated Receptor $\alpha$
}

Jungyeob Ham, Sung Jin Cho, Jaeyoung Ko, Jungwook Chin, and Heonjoong Kang*

Center for Marine Natural Products and Drug Discovery, School of Earth and Environmental Sciences, Seoul National University, NS-80, Seoul 151-747, Korea.

\section{Supporting Information}

General Procedure and Characterization of Compounds.................S2

Copy of ${ }^{1} \mathrm{H}$ and ${ }^{13} \mathrm{C}$ Spectra of Table 2 (entries 1-12)...................S11

Copy of ${ }^{1} \mathrm{H}$ and ${ }^{13} \mathrm{C}$ Spectra of Table 3 (entries 1-4).....................S35

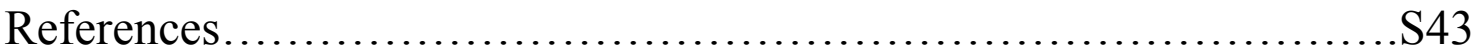


General Considerations: ${ }^{1} \mathrm{H}$ and ${ }^{13} \mathrm{C}$ NMR spectra were recorded on $300 \mathrm{MHz}$ and 500 $\mathrm{MHz}$ instruments in $\mathrm{CDCl}_{3}$ or DMSO- $d_{6}$, using TMS as the internal standard. Mass spectra were recorded by the electron impact method $(70 \mathrm{eV})$ or chemical ionization method. Commercially obtained anhydrous THF and reagents were used without further purification.

\section{General Procedure for the Formation of Alkyl Aminoaryl Sulfides (Table 2)}

To a solution of halo anilines $(2.0 \mathrm{mmol})$ in anhydrous THF $(30 \mathrm{~mL})$, a solution of ${ }^{i} \mathrm{PrMgCl}(2.0 \mathrm{M}$ solution in diethyl ether, $4.0 \mathrm{mmol})$ was added at $0{ }^{\circ} \mathrm{C}$ under $\mathrm{N}_{2}$ atmosphere and a solution of $t$ - BuLi $(1.7 \mathrm{M}$ solution in pentane, $4.0 \mathrm{mmol})$ was slowly added for $15 \mathrm{~min}$ at $-78{ }^{\circ} \mathrm{C}$. After $30 \mathrm{~min}$, sulfur powder $(2.0 \mathrm{mmol})$ was then added and the reaction mixture was slowly warmed up to room temperature for $30 \mathrm{~min}$. Finally, alkyl halide $(2.0 \mathrm{mmol})$ was added at $0{ }^{\circ} \mathrm{C}$. The reaction was monitored by thin layer chromatography. After the reaction was complete, it was quenched with aqueous $\mathrm{NH}_{4} \mathrm{Cl}$ $(35 \mathrm{~mL})$. The organic layer was separated and then the aqueous layer was extracted with EtOAc $(3 \times 30 \mathrm{~mL})$. The combined organic layer was washed with water, dried $\left(\mathrm{MgSO}_{4}\right)$, filtrated, and evaporated under reduced pressure to give the crude product. The crude compound was purified by chromatography on silica gel to obtain the desired product.

2-Benzylsulfanyl-phenylamine (Table 2, entry 1): The title compound was prepared from 2-haloaniline $(2.0 \mathrm{mmol})$ and benzyl bromide $(2.0 \mathrm{mmol})$. Flash chromatography (eluent: hexane/EtOAc $=3 / 1$ ) gave the pure product as a light brown solid (for 2iodoaniline, $319 \mathrm{mg}, 74 \%$ and for 2-bromoaniline, $284 \mathrm{mg}, 66 \%$ ). mp $37-38{ }^{\circ} \mathrm{C} .{ }^{1} \mathrm{H}$ NMR (300 MHz, $\left.\mathrm{CDCl}_{3}\right): \delta$ 7.24-7.19 (m, 3H), 7.17-7.07 (m, 4H), $6.68(\mathrm{dd}, 1 \mathrm{H}, J=8.0$, 
$1.2 \mathrm{~Hz}), 6.61(\mathrm{td}, 1 \mathrm{H}, J=7.6,1.2), 4.23$ (br s, $2 \mathrm{H}), 3.89$ (s, 2H). ${ }^{13} \mathrm{C}$ NMR $(75.5 \mathrm{MHz}$, $\left.\mathrm{CDCl}_{3}\right): \delta 148.8,138.5,136.7,130.2,129.1,128.5,127.2,118.6,115.1,39.8 . \mathrm{HRMS}$ (EI): calcd. for $\mathrm{C}_{13} \mathrm{H}_{13} \mathrm{NS}\left(\mathrm{M}^{+}\right), 215.0769$; found, 215.0769.

3-Benzylsulfanyl-phenylamine (Table 2, entry 2): The title compound was prepared from 3-haloaniline $(2.0 \mathrm{mmol})$ and benzyl bromide $(2.0 \mathrm{mmol})$. Flash chromatography (eluent: hexane/EtOAc $=3 / 1$ ) gave the pure product as a brown solid (for 3-iodoaniline, $357 \mathrm{mg}, 83 \%$ and for 3-bromoaniline, $349 \mathrm{mg}, 81 \%$ ) $\mathrm{mp} 45-47{ }^{\circ} \mathrm{C}$. ${ }^{1} \mathrm{H}$ NMR $\left(300 \mathrm{MHz}, \mathrm{CDCl}_{3}\right): \delta$ 7.30-7.22 (m, 5H), $7.03(\mathrm{t}, 1 \mathrm{H}), 6.70(\mathrm{dt}, 1 \mathrm{H}), 6.62(\mathrm{t}$, 1H), $6.48(\mathrm{dt}, 1 \mathrm{H}), 4.09$ (s, 2H), $3.60(\mathrm{bs}, 2 \mathrm{H}) .{ }^{13} \mathrm{C} \mathrm{NMR}\left(75.5 \mathrm{MHz}, \mathrm{CDCl}_{3}\right): \delta$ 147.0, 137.8, 137.6, 129.8, 129.0, 128.7, 127.3, 119.7, 116.0, 113.4, 38.9. HRMS (EI): calcd. for $\mathrm{C}_{13} \mathrm{H}_{13} \mathrm{NS}\left(\mathrm{M}^{+}\right), 215.0769$; found, 215.0770.

4-Benzylsulfanyl-phenylamine (Table 2, entry 3): The title compound was prepared from 4-haloaniline $(2.0 \mathrm{mmol})$ and benzyl bromide $(2.0 \mathrm{mmol})$. Flash chromatography (eluent: hexane/EtOAc $=3 / 1$ ) gave the pure product as a brown oil (for 4-iodoaniline, $405 \mathrm{mg}, 94 \%$ and for 4-bromoaniline, $379 \mathrm{mg}, 88 \%) .{ }^{1} \mathrm{H}$ NMR (300 MHz, $\left.\mathrm{CDCl}_{3}\right): \delta$ 7.27-7.08 (m, 7H), $6.54(\mathrm{dt}, 2 \mathrm{H}), 3.92$ (s, 2H), 3.66 (br s, 2H). ${ }^{13} \mathrm{C}$ NMR $(75.5 \mathrm{MHz}$, $\left.\mathrm{CDCl}_{3}\right): \delta 146.4,138.6,134.9,129.1,128.5,127.0,123.1,115.6,41.9 . \mathrm{HRMS}$ (EI): calcd. for $\mathrm{C}_{13} \mathrm{H}_{13} \mathrm{NS}\left(\mathrm{M}^{+}\right), 215.0769$; found, 215.0769.

4-Benzylsulfanyl-2-bromo-phenylamine (Table 2, entry 4): The title compound was prepared from 2,4-dibromoaniline $(2.0 \mathrm{mmol})$ and benzyl bromide $(2.0 \mathrm{mmol})$. Flash chromatography (eluent: hexane/EtOAc $=3 / 1$ ) gave the pure product as a yellow 
oil (435 mg, 74\%). ${ }^{1} \mathrm{H}$ NMR (300 MHz, $\left.\mathrm{CDCl}_{3}\right): \delta 7.39(\mathrm{~d}, 1 \mathrm{H}), 7.29-7.12(\mathrm{~m}, 5 \mathrm{H})$, $7.04(\mathrm{dd}, 1 \mathrm{H}), 6.61$ (d, 1H), 4.11 (br s, $2 \mathrm{H}), 3.93$ (s, 2H). ${ }^{13} \mathrm{C} \mathrm{NMR}\left(75.5 \mathrm{MHz}, \mathrm{CDCl}_{3}\right)$ : $\delta 144.2,138.4,137.6,134.0,129.4,128.8,127.5,124.5,116.0,109.3,42.1$. HRMS (EI): calcd. for $\mathrm{C}_{13} \mathrm{H}_{12} \mathrm{NS}^{81} \mathrm{Br}\left(\mathrm{M}^{+}\right)$, 294.9853; found, 294.9853 .

4-Benzylsulfanyl-2-methyl-phenylamine (Table 2, entry 5): The title compound was prepared from 4-iodo-2-methylaniline $(2.0 \mathrm{mmol})$ and benzyl bromide $(2.0$ mmol). Flash chromatography (eluent: hexane/EtOAc $=3 / 1$ ) gave the pure product as a clear oil (394 mg, 86\%). ${ }^{1} \mathrm{H}$ NMR (300 MHz, $\left.\mathrm{CDCl}_{3}\right): \delta 7.32-7.24(\mathrm{~m}, 5 \mathrm{H}), 7.16(\mathrm{t}, 1 \mathrm{H})$, $6.86(\mathrm{~d}, 1 \mathrm{H}), 6.82(\mathrm{t}, 1 \mathrm{H}), 6.65(\mathrm{dd}, 1 \mathrm{H}), 4.11(\mathrm{~s}, 2 \mathrm{H}), 3.73(\mathrm{~s}, 3 \mathrm{H}) .{ }^{13} \mathrm{C}$ NMR $(75.5$ $\left.\mathrm{MHz}, \mathrm{CDCl}_{3}\right): \delta 160.1,138.1,137.8,130.0,129.2,128.9,127.6,122.1,115.2,112.6$, 55.6, 39.2. HRMS (EI): calcd. for $\mathrm{C}_{14} \mathrm{H}_{15} \mathrm{NS}\left(\mathrm{M}^{+}\right)$, 229.0925; found, 229.0921 .

4-(4-Aminophenylsulfanyl)-benzonitrile (Table 2, entry 6): The title compound was prepared from 4-iodoaniline $(2.0 \mathrm{mmol})$ and 4-(bromomethyl)benzonitrile $(2.0$ $\mathrm{mmol}$ ). Flash chromatography (eluent: hexane/EtOAc $=3 / 1$ ) gave the pure product as a yellow solid, mp 130-132 ${ }^{\circ} \mathrm{C}(428 \mathrm{mg}, 89 \%) .{ }^{1} \mathrm{H}$ NMR $\left(300 \mathrm{MHz}, \mathrm{CDCl}_{3}\right): \delta 7.50(\mathrm{dt}$, 2H), 7.19 (d, 2H), 7.05 (dt, 2H), $6.54(\mathrm{dt}, 2 \mathrm{H}), 3.89$ (s, 2H), 3.75 (br s, 2H). ${ }^{13} \mathrm{C}$ NMR $\left(75.5 \mathrm{MHz}, \mathrm{CDCl}_{3}\right): \delta 147.0,144.5,135.6,132.2,129.8,121.3,119.1,115.6,110.7$, 41.8. HRMS (EI): calcd. for $\mathrm{C}_{14} \mathrm{H}_{12} \mathrm{~N}_{2} \mathrm{~S}\left(\mathrm{M}^{+}\right), 240.0721$; found, 240.0721 .

4-(2-Methyl-allylsulfanyl)-phenylamine (Table 2, entry 7): The title compound was prepared from 4-iodoaniline $(2.0 \mathrm{mmol})$ and 3-bromo-2-methylprop-1-ene $(2.0$ $\mathrm{mmol}$ ). Flash chromatography (eluent: hexane/EtOAc $=3 / 1$ ) gave the pure product as a 
dark brown oil (333 mg, 93\%). ${ }^{1} \mathrm{H}$ NMR (300 MHz, $\left.\mathrm{CDCl}_{3}\right): \delta 7.21$ (dt, 2H), 6.59 (dt, 2H), 4.67 (m, 2H), 3.65 (br s, 2H), 3.35 (s, 2H), 1.83 (s, 3H). ${ }^{13} \mathrm{C}$ NMR (75.5 MHz, $\left.\mathrm{CDCl}_{3}\right): \delta 146.2,141.6,134.7,123.5,115.6,113.8,44.8,21.1$. HRMS (EI): calcd. for $\mathrm{C}_{10} \mathrm{H}_{13} \mathrm{NS}\left(\mathrm{M}^{+}\right)$179.0769; found 179.0768.

2-(4-Amino-phenylsulfanyl)-1-phenyl-ethanone (Table 2, entry 8): The title compound was prepared from 4-iodoaniline $(2.0 \mathrm{mmol})$ and 2-bromo-1-phenylethanone $(2.0 \mathrm{mmol})$. Flash chromatography (eluent: hexane/EtOAc $=3 / 1)$ gave the pure product as a brown solid, mp 103-106 ${ }^{\circ} \mathrm{C}(448 \mathrm{mg}, 92 \%) .{ }^{1} \mathrm{H}$ NMR $\left(300 \mathrm{MHz}, \mathrm{CDCl}_{3}\right): \delta 7.91$ (m, 2H), $7.56(\mathrm{~m}, 1 \mathrm{H}), 7.44(\mathrm{~m}, 2 \mathrm{H}), 7.22(\mathrm{dt}, 2 \mathrm{H}), 6.57(\mathrm{dt}, 2 \mathrm{H}), 4.07(\mathrm{~s}, 2 \mathrm{H}), 3.76(\mathrm{br} \mathrm{s}$, 2H). ${ }^{13} \mathrm{C}$ NMR $\left(75.5 \mathrm{MHz}, \mathrm{CDCl}_{3}\right): \delta 194.7,147.1,135.8,135.5,133.4,129.0,128.8$, 121.5, 115.8, 43.5. HRMS (EI): calcd. for $\mathrm{C}_{14} \mathrm{H}_{13} \mathrm{NOS}\left(\mathrm{M}^{+}\right)$, 243.0718; found, 243.0719.

(4-Amino-phenylsulfanyl)-acetic acid tert-butyl ester (Table 2, entry 9): The title compound was prepared from 4-iodoaniline $(2.0 \mathrm{mmol})$ and tert-butyl 2-bromoacetate $(2.0 \mathrm{mmol})$. Flash chromatography (eluent: hexane/EtOAc $=3 / 1)$ gave the pure product as a yellow oil (454 mg, 95\%). ${ }^{1} \mathrm{H}$ NMR $\left(300 \mathrm{MHz}, \mathrm{CDCl}_{3}\right): \delta 7.29(\mathrm{dt}, 2 \mathrm{H}), 6.60(\mathrm{dt}$, 2H), 3.74 (br s, 2H), 3.37 (s, 2H), $1.40(\mathrm{~s}, 9 \mathrm{H}) .{ }^{13} \mathrm{C} \mathrm{NMR}\left(75.5 \mathrm{MHz}, \mathrm{CDCl}_{3}\right): \delta$ 169.7, 147.0, 135.0, 122.5, 115.8, 81.8, 40.6, 28.3. HRMS (EI): calcd,. for $\mathrm{C}_{12} \mathrm{H}_{17} \mathrm{NO}_{2} \mathrm{~S}\left(\mathrm{M}^{+}\right)$, 239.0980; found, 239.0980.

4-(5-Phenyl-pentylsulfanyl)-phenylamine (Table 2, entry 10): The title compound was prepared from 4-iodoaniline $(2.0 \mathrm{mmol})$ and 1-(5-bromopentyl $)$ benzene $(2.0 \mathrm{mmol})$. Flash chromatography (eluent: hexane/EtOAc $=3 / 1$ ) gave the pure product as a clear oil 
(456 mg, 84\%). ${ }^{1} \mathrm{H}$ NMR (300 MHz, $\left.\mathrm{CDCl}_{3}\right): \delta$ 7.29-7.11 (m, 7H), $6.60(\mathrm{dt}, 2 \mathrm{H}), 3.50$ (br s, 2H), $2.75(\mathrm{t}, 2 \mathrm{H}), 2.58(\mathrm{t}, 2 \mathrm{H}), 1.62-1.53(\mathrm{~m}, 4 \mathrm{H}), 1.47-1.39(\mathrm{~m}, 2 \mathrm{H}) .{ }^{13} \mathrm{C} \mathrm{NMR}$ $\left(75.5 \mathrm{MHz}, \mathrm{CDCl}_{3}\right): \delta 145.9,142.8,134.0,129.5,128.6,128.4,125.8,115.8,36.5,36.0$, 31.2, 29.5, 28.5. HRMS (EI): calcd. for $\mathrm{C}_{17} \mathrm{H}_{21} \mathrm{NS}\left(\mathrm{M}^{+}\right)$, 271.1395; found, 271.1393.

4-Cyclohexylmethylsulfanyl-phenylamine (Table 2, entry 11): The title compound was prepared from 4-iodoaniline $(2.0 \mathrm{mmol})$ and (bromomethyl)cyclohexane $(2.0$ mmol). Flash chromatography (eluent: hexane/EtOAc $=3 / 1$ ) gave the pure product as a clear oil (359 mg, 81\%). ${ }^{1} \mathrm{H}$ NMR (300 MHz, $\left.\mathrm{CDCl}_{3}\right): \delta 7.42(\mathrm{~d}, 2 \mathrm{H}, J=8.8 \mathrm{~Hz}), 7.21$ (dt, 2H), $6.60(\mathrm{dt}, 2 \mathrm{H}), 3.66$ (br s, 2H), $2.67(\mathrm{~d}, 2 \mathrm{H}), 1.86(\mathrm{~d}, 2 \mathrm{H}), 1.76-1.57(\mathrm{~m}, 3 \mathrm{H})$, 1.54-1.36 (m, 1H), 1.30-1.08 (m, 3H), 1.02-0.85 (m, 2H). ${ }^{13} \mathrm{C}$ NMR (75.5 MHz, $\left.\mathrm{CDCl}_{3}\right): \delta 145.7,139.0,133.5,115.8,115.1,44.1,37.7,32.9,26.6,26.3$. HRMS (EI): calcd. for $\mathrm{C}_{13} \mathrm{H}_{19} \mathrm{NS}\left(\mathrm{M}^{+}\right), 221.1238$; found, 221.1242.

1-(4-Aminophenylsulfanyl)-hex-5-en-2-ol (Table 2, entry 12): The title compound was prepared from 4-iodoaniline $(2.0 \mathrm{mmol})$ and 2-(but-3-enyl)oxirane $(2.0 \mathrm{mmol})$. Flash chromatography (eluent: hexane/EtOAc $=3 / 1$ ) gave the pure product as a yellow oil (389 mg, 87\%). ${ }^{1} \mathrm{H}$ NMR (300 MHz, $\left.\mathrm{CDCl}_{3}\right): \delta 7.26$ (dt, 2H), 6.61 (dt, 2H), 5.79 (m, 1H), $4.98(\mathrm{~m}, 2 \mathrm{H}), 3.73$ (br s, 2H), $3.59(\mathrm{~m}, 1 \mathrm{H}), 2.97$ (dd, 1H), $2.68(\mathrm{dd}, 1 \mathrm{H}), 2.59$ (bs, 1H), 2.24-2.05 (m, 2H), 1.66-1.50 (m, 3H). $\left.{ }^{13} \mathrm{C} \mathrm{NMR} \mathrm{(75.5} \mathrm{MHz,} \mathrm{CDCl}_{3}\right): \delta$ 146.6, 138.4, 134.6, 122.1, 115.9, 115.0, 68.7, 44.9, 35.2, 30.2. HRMS (EI): calcd. for $\mathrm{C}_{12} \mathrm{H}_{17} \mathrm{NOS}\left(\mathrm{M}^{+}\right), 223.1031$; found, 223.1032.

General Procedure for the Formation of Alkyl (Alkylamino)aryl Sulfides (Table 3) 
To a solution of 4-bromobenzylamine hydrochloride or 4-bromophenethylamine (2.0 mmol) in anhydrous THF $(30 \mathrm{~mL})$, a solution of ${ }^{i} \mathrm{PrMgCl}(2.0 \mathrm{M}$ solution in diethyl ether, $4.0 \mathrm{mmol}$ ) was added at $0{ }^{\circ} \mathrm{C}$ under $\mathrm{N}_{2}$ atmosphere and then a solution of $t$-BuLi (1.7 $\mathrm{N}$ solution in pentane, $4.0 \mathrm{mmol}$ ) was slowly added for $15 \mathrm{~min}$ at $-78{ }^{\circ} \mathrm{C}$. After 30 min, sulfur powder $(2.0 \mathrm{mmol})$ was added and the reaction mixture was slowly warmed up to room temperature for $30 \mathrm{~min}$. Finally, alkyl halide $(2.0 \mathrm{mmol})$ was slowly added. The reaction was monitored by thin layer chromatography. After the reaction was completed, the reaction mixture was quenched with aqueous $\mathrm{NH}_{4} \mathrm{Cl}(35 \mathrm{~mL})$. The organic layer was separated and then the aqueous layer was extracted with EtOAc $(3 \times 30$ $\mathrm{mL})$. The combined extract was washed with water, dried $\left(\mathrm{MgSO}_{4}\right)$, filtrated, and evaporated under reduced pressure to give the crude product. The crude compound was purified by chromatography on silica gel to obtain the desired compound.

4-Benzylsulfanyl-benzylamine (Table 3, entry 1): The title compound was prepared from 4-bromobenzylamine hydrochloride $(2.0 \mathrm{mmol})$ and benzyl bromide $(2.0 \mathrm{mmol})$. Flash chromatography (eluent: $5 \% \mathrm{MeOH}$ in $\mathrm{CH}_{2} \mathrm{Cl}_{2}$ ) gave the pure product as a white solid, mp 95-98 ${ }^{\circ} \mathrm{C}(440 \mathrm{mg}, 96 \%) .{ }^{1} \mathrm{H}$ NMR (300 MHz, $\left.\mathrm{CDCl}_{3}\right): \delta$ 7.29-7.18 (m, 9H), 4.09 (s, 2H), $3.81(\mathrm{~s}, 2 \mathrm{H}) .{ }^{13} \mathrm{C} \mathrm{NMR}\left(75.5 \mathrm{MHz}, \mathrm{CDCl}_{3}\right): \delta 141.9,137.8,134.6,130.6$, 129.0, 128.7, 127.8, 127.3, 46.2, 39.6. HRMS (EI): calcd. for $\mathrm{C}_{14} \mathrm{H}_{15} \mathrm{NS}\left(\mathrm{M}^{+}\right)$, 229.0925; found, 229.0928.

2-(4-Benzylsulfanyl-phenyl)-ethylamine (Table 3, entry 2): The title compound was prepared from 4-bromophenethylamine $(2.0 \mathrm{mmol})$ and benzyl bromide $(2.0 \mathrm{mmol})$. Flash chromatography (eluent: $5 \% \mathrm{MeOH}$ in $\mathrm{CH}_{2} \mathrm{Cl}_{2}$ ) gave the pure product as a brown 
solid, mp 98-101 ${ }^{\circ} \mathrm{C}(477 \mathrm{mg}, 98 \%) .{ }^{1} \mathrm{H} \mathrm{NMR}\left(300 \mathrm{MHz}, \mathrm{CDCl}_{3}\right): \delta$ 7.28-7.21 (m, 7H), $7.09(\mathrm{~d}, 2 \mathrm{H}), 4.09(\mathrm{~s}, 2 \mathrm{H}), 2.94(\mathrm{t}, 2 \mathrm{H}), 2.70(\mathrm{t}, 2 \mathrm{H}), 1.75$ (br s, 2H). ${ }^{13} \mathrm{C}$ NMR $(75.5$ $\left.\mathrm{MHz}, \mathrm{CDCl}_{3}\right): \delta 138.4,137.8,134.0,130.7,129.6,129.0,128.7,127.3,43.5,39.7,29.9$. HRMS (EI): calcd. for $\mathrm{C}_{15} \mathrm{H}_{17} \mathrm{NS}\left(\mathrm{M}^{+}\right)$, 243.1082; found, 243.1083.

tert-Butyl [4-(2-aminoethyl)-phenylsulfanyl]-acetate (Table 3, entry 3): The title compound was prepared from 4-bromophenethylamine $(2.0 \mathrm{mmol})$ and tert-butyl 2bromoacetate $(2.0 \mathrm{mmol})$. Flash chromatography (eluent: $5 \% \mathrm{MeOH}$ in $\mathrm{CH}_{2} \mathrm{Cl}_{2}$ ) gave the pure product as a yellow oil (503 mg, 94\%). ${ }^{1} \mathrm{H}$ NMR (300 MHz, $\left.\mathrm{CDCl}_{3}\right): \delta 7.32(\mathrm{~d}$, 2H), $7.17(\mathrm{~d}, 2 \mathrm{H}), 4.98(\mathrm{br} \mathrm{s}, 2 \mathrm{H}), 3.51(\mathrm{~s}, 2 \mathrm{H}), 3.18(\mathrm{t}, 2 \mathrm{H}), 3.02(\mathrm{t}, 2 \mathrm{H}), 1.40(\mathrm{~s}, 9 \mathrm{H})$ ${ }^{13} \mathrm{C}$ NMR (75.5 MHz, $\left.\mathrm{CDCl}_{3}\right): \delta 169.1,135.7,134.2,130.5,129.7,82.3,41.6,38.0$, 34.2, 28.1. HRMS (EI): calcd. for $\mathrm{C}_{14} \mathrm{H}_{21} \mathrm{NO}_{2} \mathrm{~S}\left(\mathrm{M}^{+}\right)$, 267.1293; found, 267.1294.

\section{tert-Butyl 2-[4-(2-aminoethyl)-phenylsulfanyl]-2-methylpropanoate (Table 3,} entry 4): To a solution of 4-bromophenethylamine (400.2 $\mathrm{mg}, 2.0 \mathrm{mmol})$ in anhydrous THF (30 mL) was slowly added ${ }^{i} \mathrm{PrMgCl}(2.0 \mathrm{M}$ solution in diethyl ether, $2.0 \mathrm{~mL}, 4.0$ $\mathrm{mmol}$ ) at $0{ }^{\circ} \mathrm{C}$ for $10 \mathrm{~min}$ under $\mathrm{N}_{2}$. After $30 \mathrm{~min}, \mathrm{t}$-BuLi (1.7 M solution in pentane, 2.4 $\mathrm{mL}, 4.0 \mathrm{mmol}$ ) was slowly added at $-78^{\circ} \mathrm{C}$ for $20 \mathrm{~min}$ and the reaction mixture was stirred for an additional $30 \mathrm{~min}$. Sulfur powder $(64 \mathrm{mg}, 2.0 \mathrm{mmol})$ was added at once and the reaction mixture was slowly warmed up to room temperature for $30 \mathrm{~min}$. After the reaction was complete, the solvent was completely removed by an evaporator under the atmosphere. To a solution of the residual product in $\mathrm{MeOH}(30 \mathrm{~mL})$ was added $\mathrm{KOH}(117.8 \mathrm{mg}, 2.1 \mathrm{mmol})$. The reaction mixture was heated at $60{ }^{\circ} \mathrm{C}$ for additional $1 \mathrm{~h}$. After that time, the reaction mixture was poured into $\mathrm{NH}_{4} \mathrm{Cl}$ solution $(35 \mathrm{~mL})$ and 
extracted with EtOAc $(3 \times 30 \mathrm{~mL})$. The combined organic layer was washed with $\mathrm{H}_{2} \mathrm{O}$, dried $\left(\mathrm{MgSO}_{4}\right)$, filtered off, and then concentrated on a rotary evaporator. The crude compound was purified by chromatography (eluent: $5 \% \mathrm{MeOH}$ in $\mathrm{CH}_{2} \mathrm{Cl}_{2}$ ) on silica gel to obtain the pure product as a white oil $(544 \mathrm{mg}, 92 \%) .{ }^{1} \mathrm{H}$ NMR $\left(300 \mathrm{MHz}, \mathrm{CDCl}_{3}\right)$ : $\delta$ $7.44(\mathrm{~d}, 7 \mathrm{H}, J=8.0 \mathrm{~Hz}), 7.16(\mathrm{~d}, 7 \mathrm{H}, J=8.0), 2.97(\mathrm{t}, 2 \mathrm{H}), 2.76(\mathrm{t}, 2 \mathrm{H}), 1.44(\mathrm{~s}, 6 \mathrm{H})$, $1.43(\mathrm{~s}, 9 \mathrm{H}) .{ }^{13} \mathrm{C}$ NMR $\left(75.5 \mathrm{MHz}, \mathrm{CDCl}_{3}\right): \delta 173.5,141.5,137.4,129.4,81.3,51.7$, 43.8, 40.2, 28.3, 26.5. HRMS (EI): calcd. for $\mathrm{C}_{16} \mathrm{H}_{25} \mathrm{NO}_{2} \mathrm{~S}\left(\mathrm{M}^{+}\right)$, 295.1606; found, 295.1605 . 


\section{${ }^{1} \mathrm{H}$ and ${ }^{13} \mathrm{C}-\mathrm{NMR}$ Spectra of Compounds}


2-Benzylsulfanyl-phenylamine (Table 2, entry 1, ref 1)

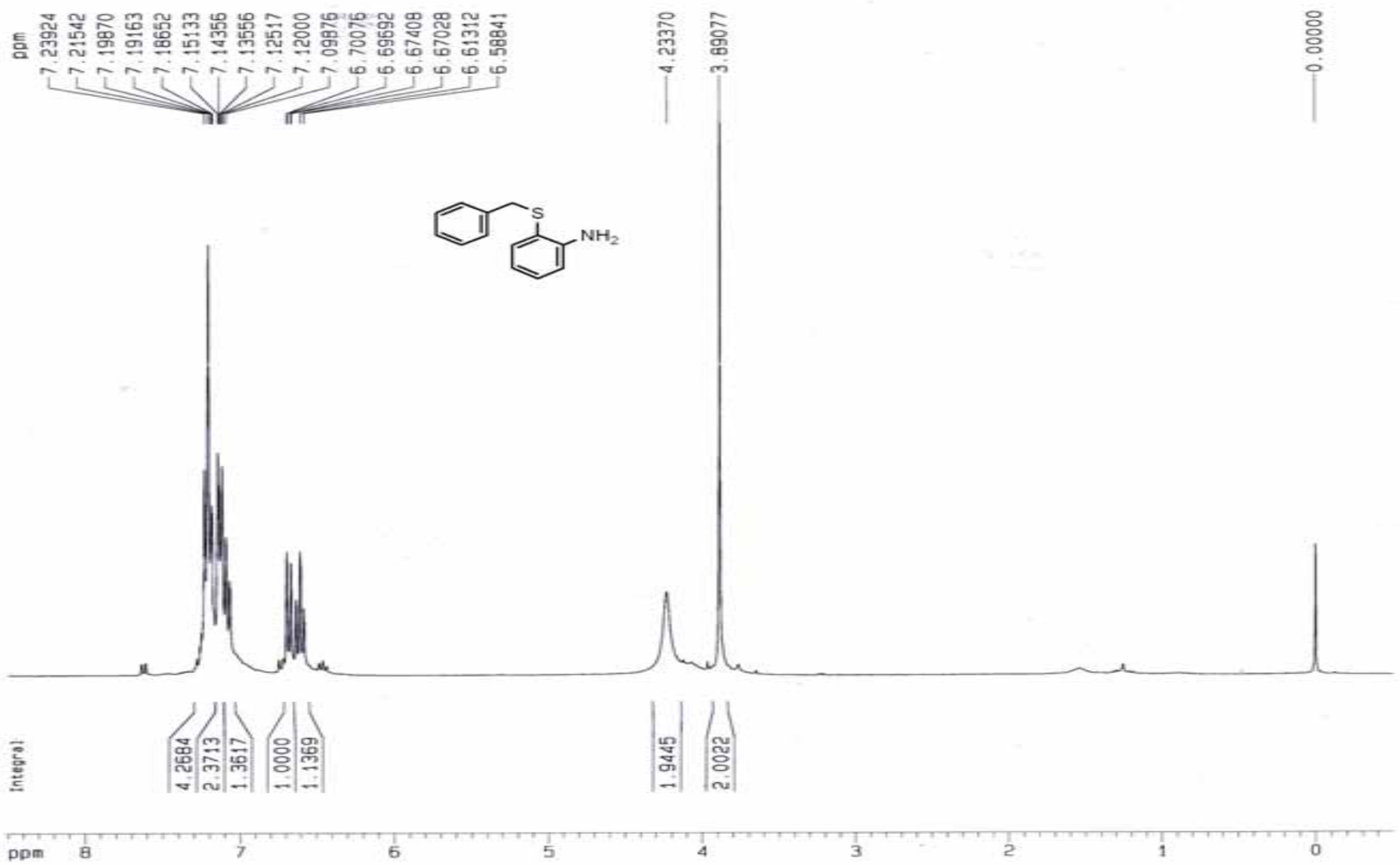




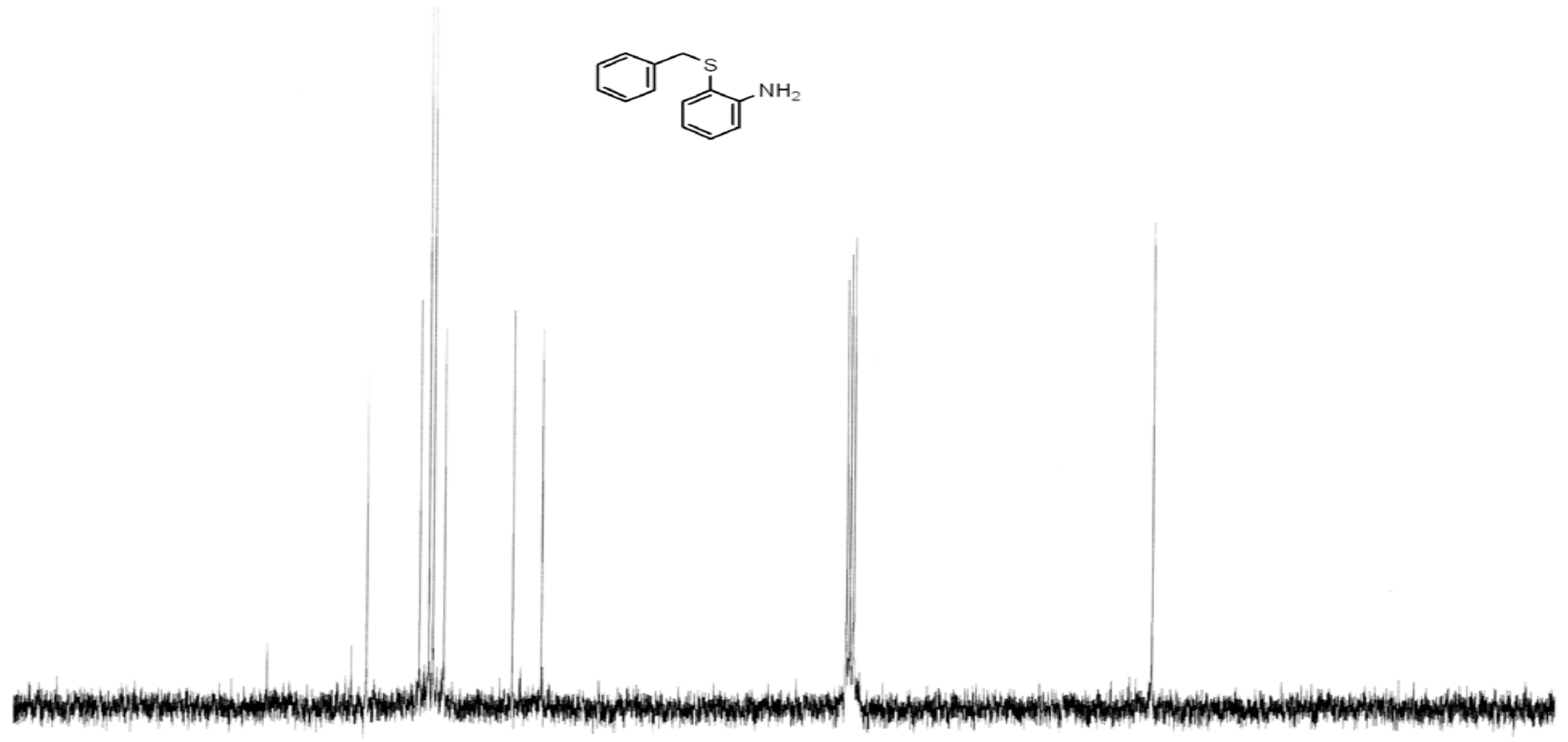


3-Benzylsulfanyl-phenylamine (Table 2, entry 2, ref 2)

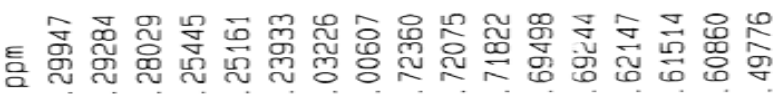
rararara $\longrightarrow$

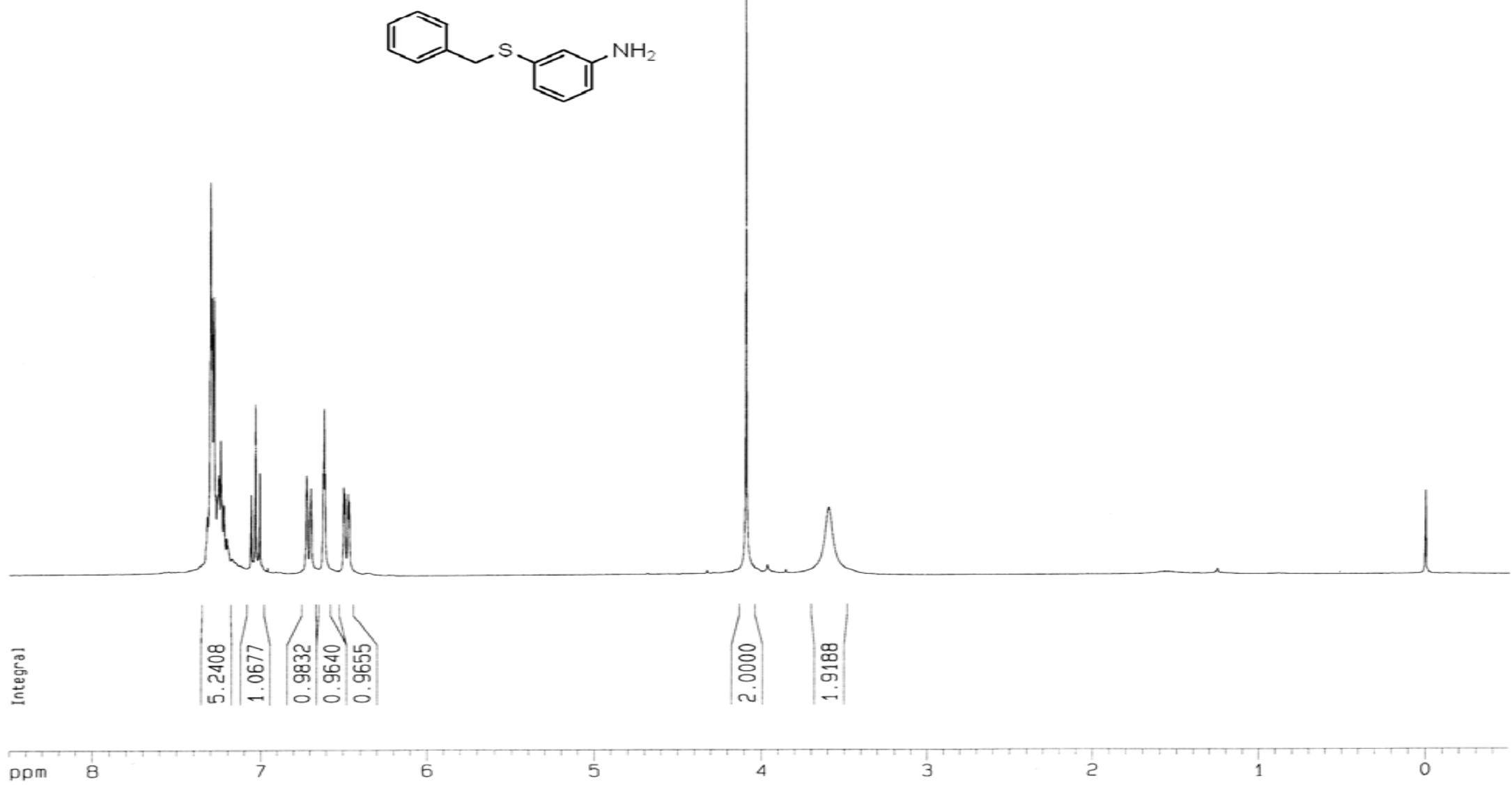




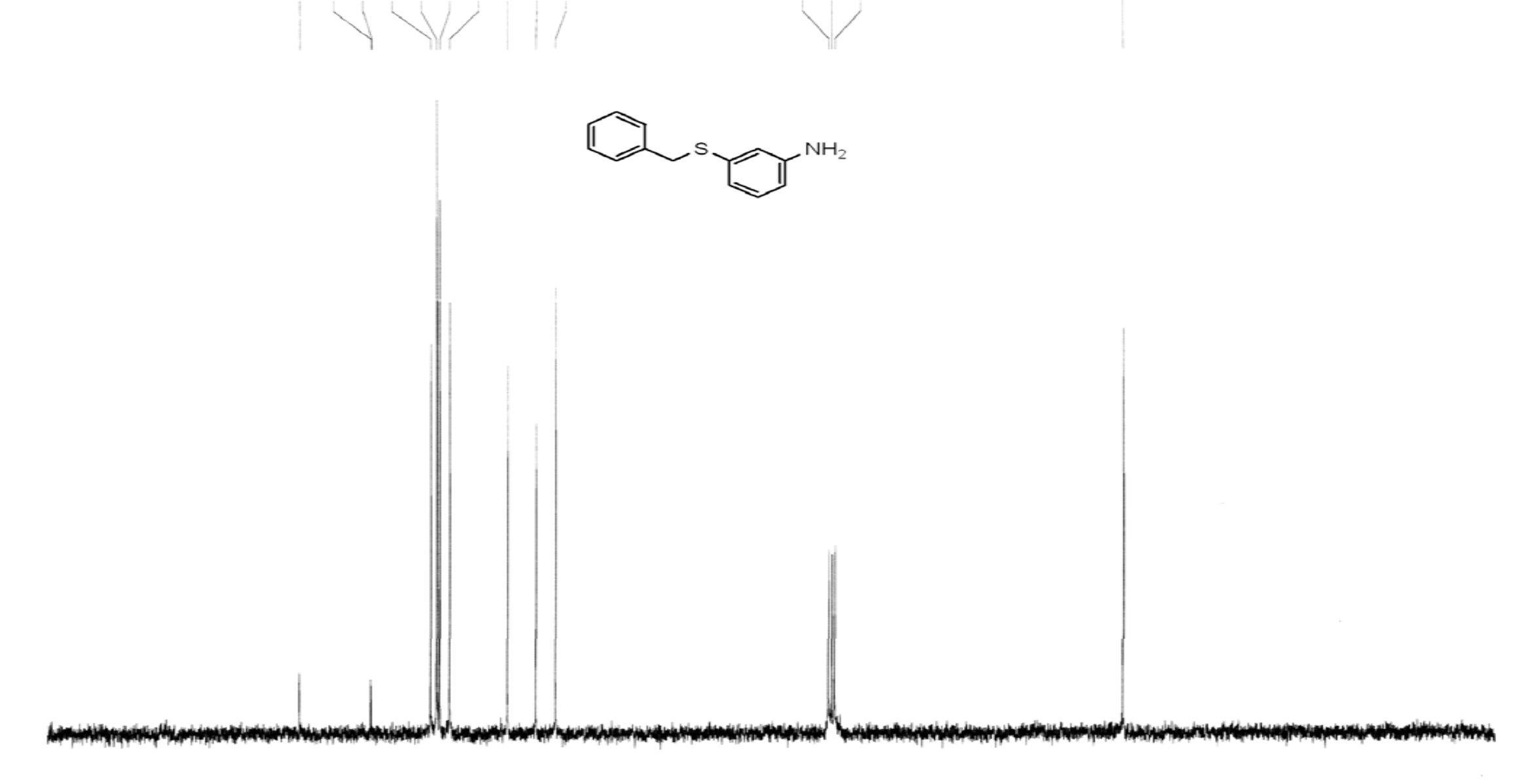


4-Benzylsulfanyl-phenylamine (Table 2, entry 3, ref 3)

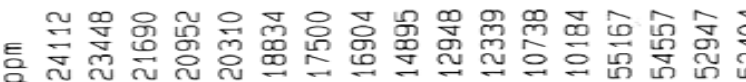

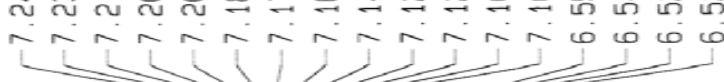

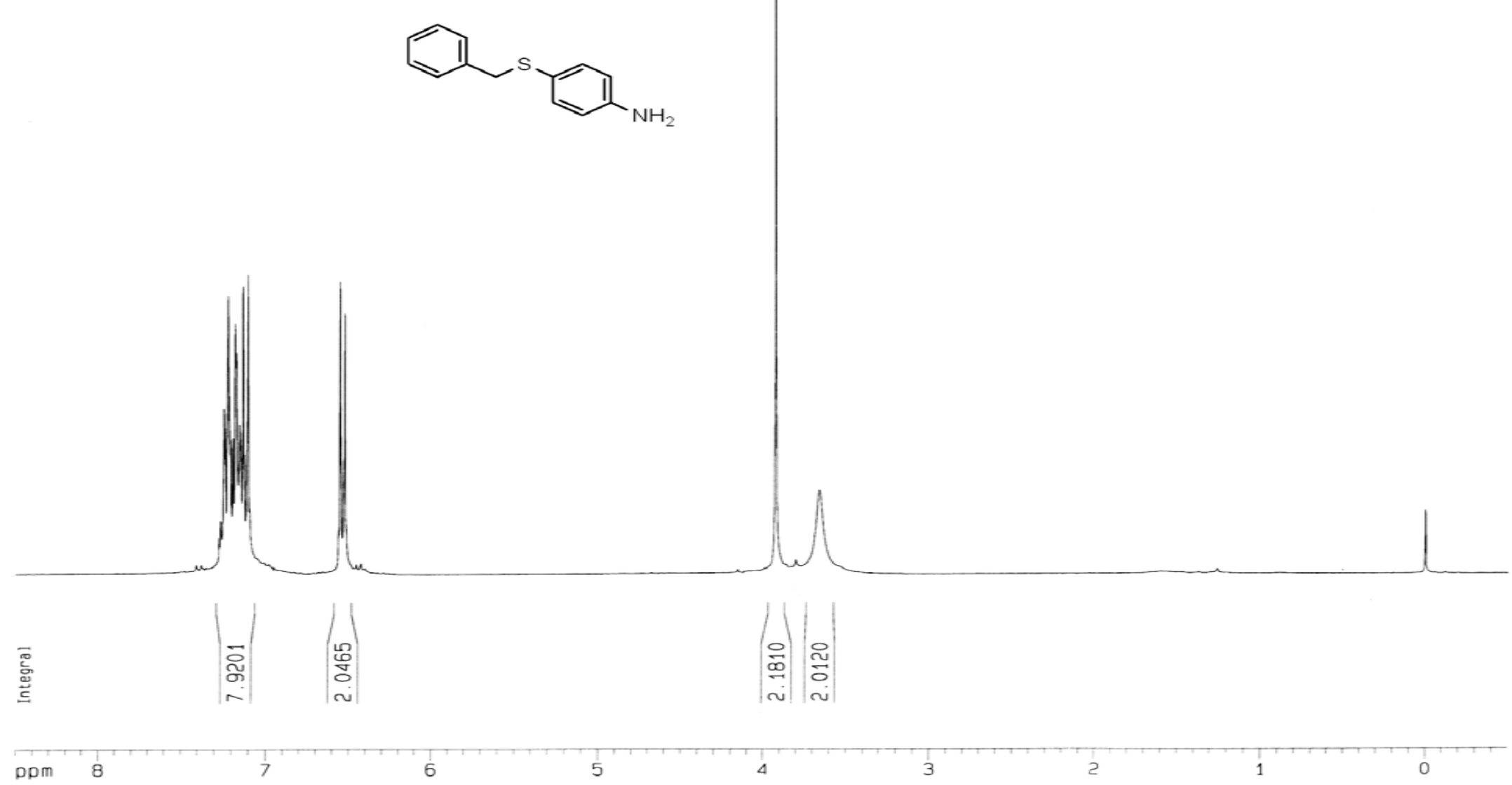

Tili

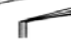




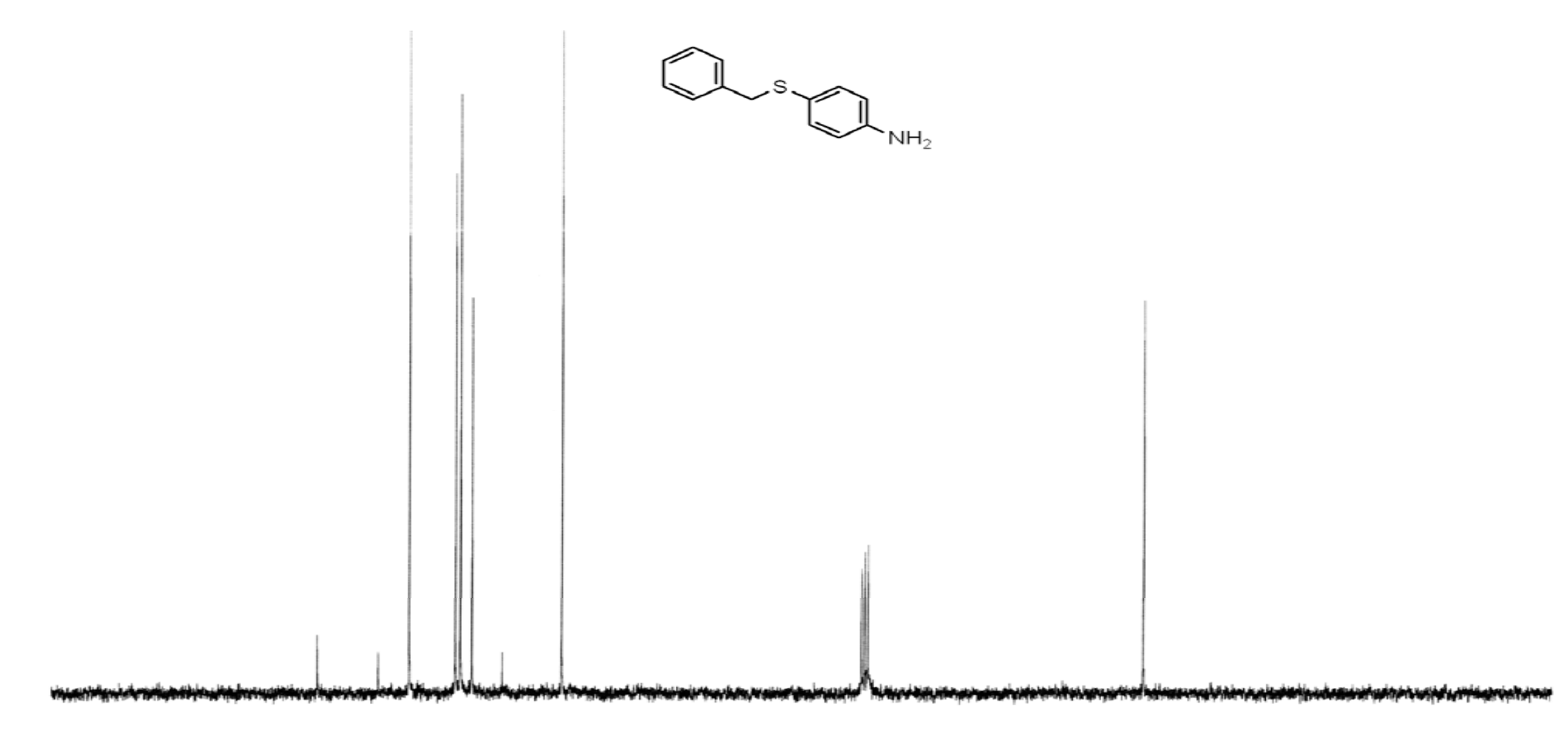


4-Benzylsulfanyl-2-bromo-phenylamine (Table 2, entry 4)

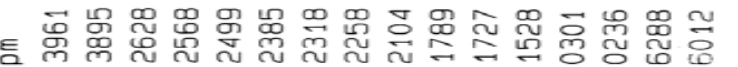

$\sim$
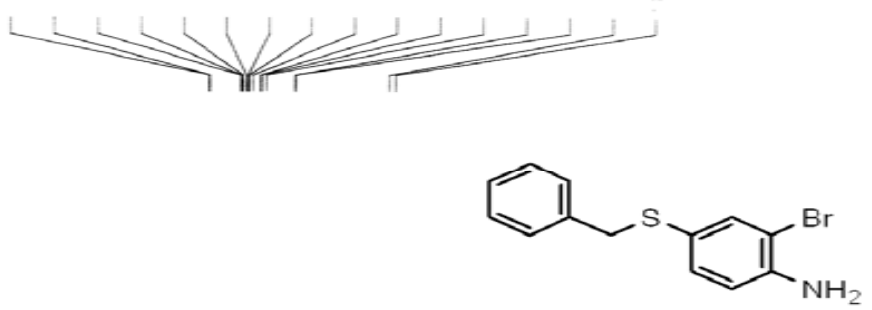

$\mathrm{NH}_{2}$

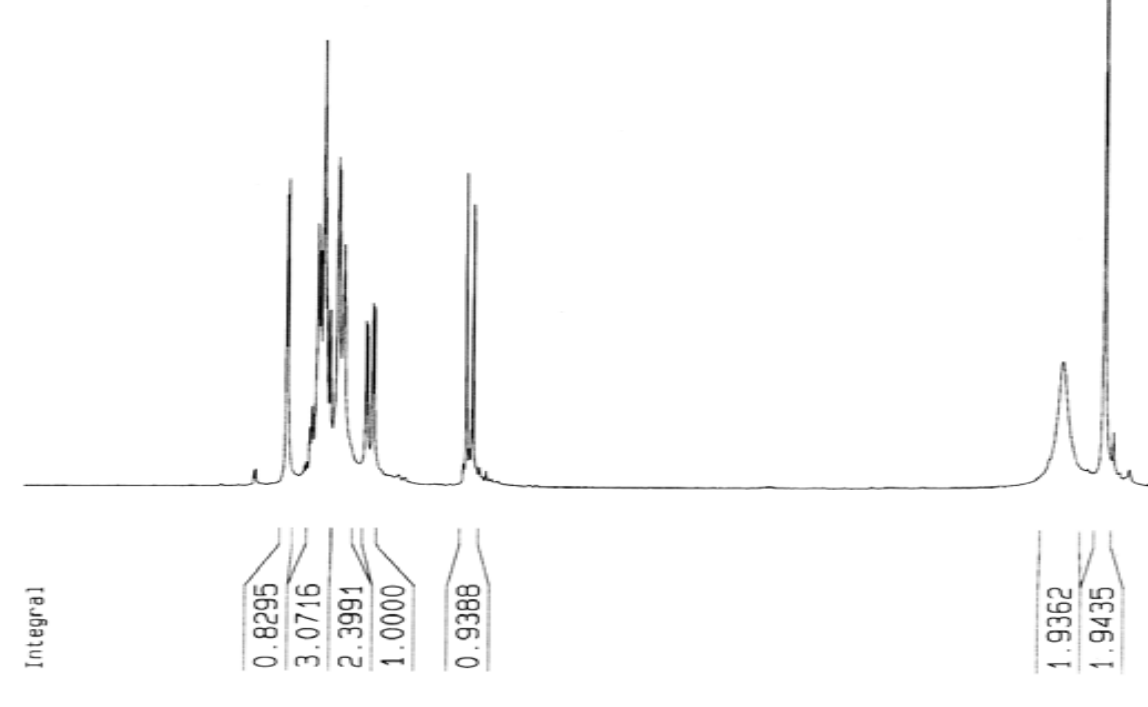

ppm 8 


\section{.}

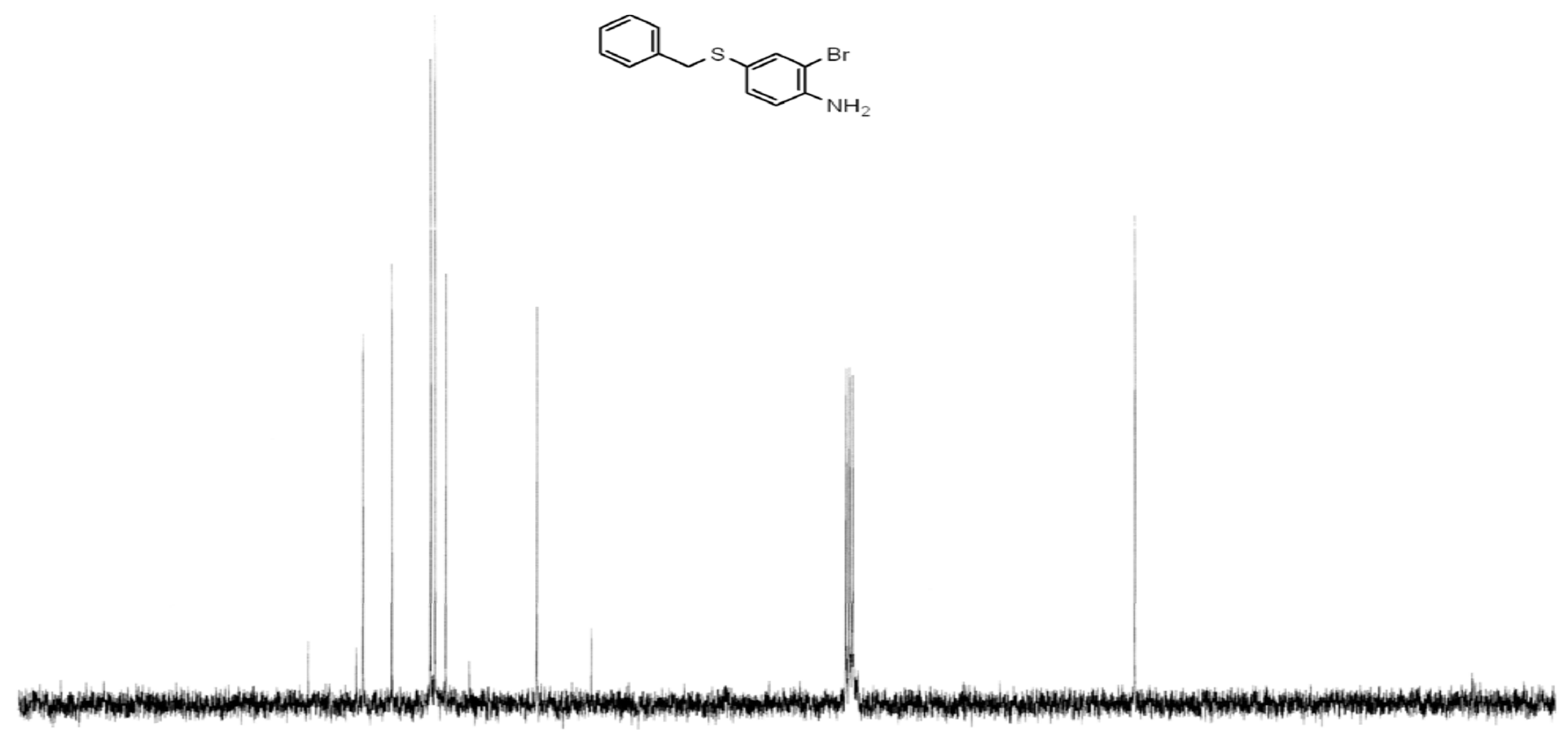


4-Benzylsulfanyl-2-methyl-phenylamine (Table 2, entry 5)

VIII-20 $20040906 / 1 \mathrm{H}$

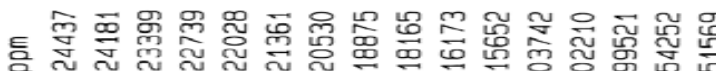

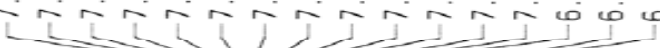

స్

峁学

호.

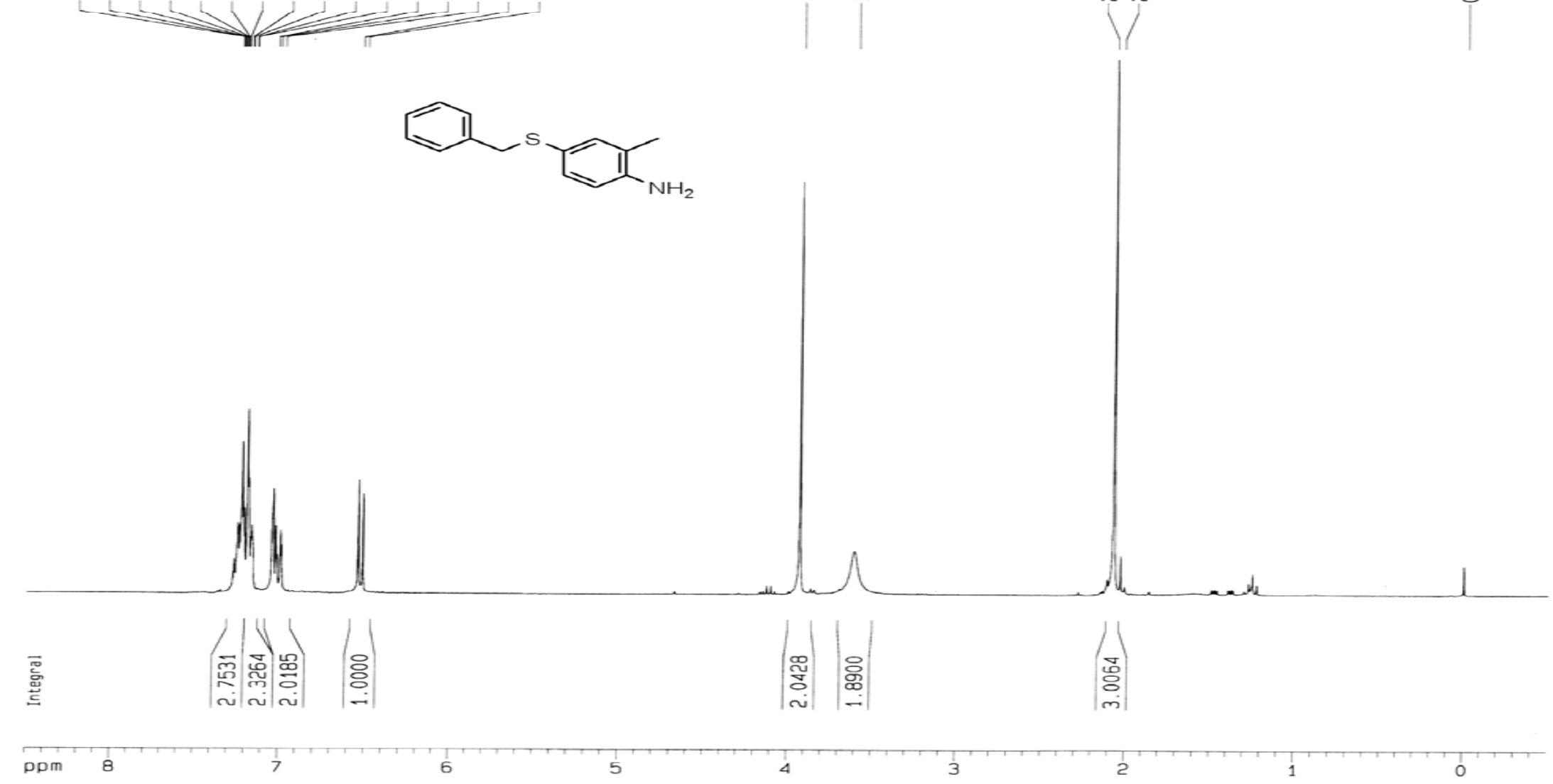




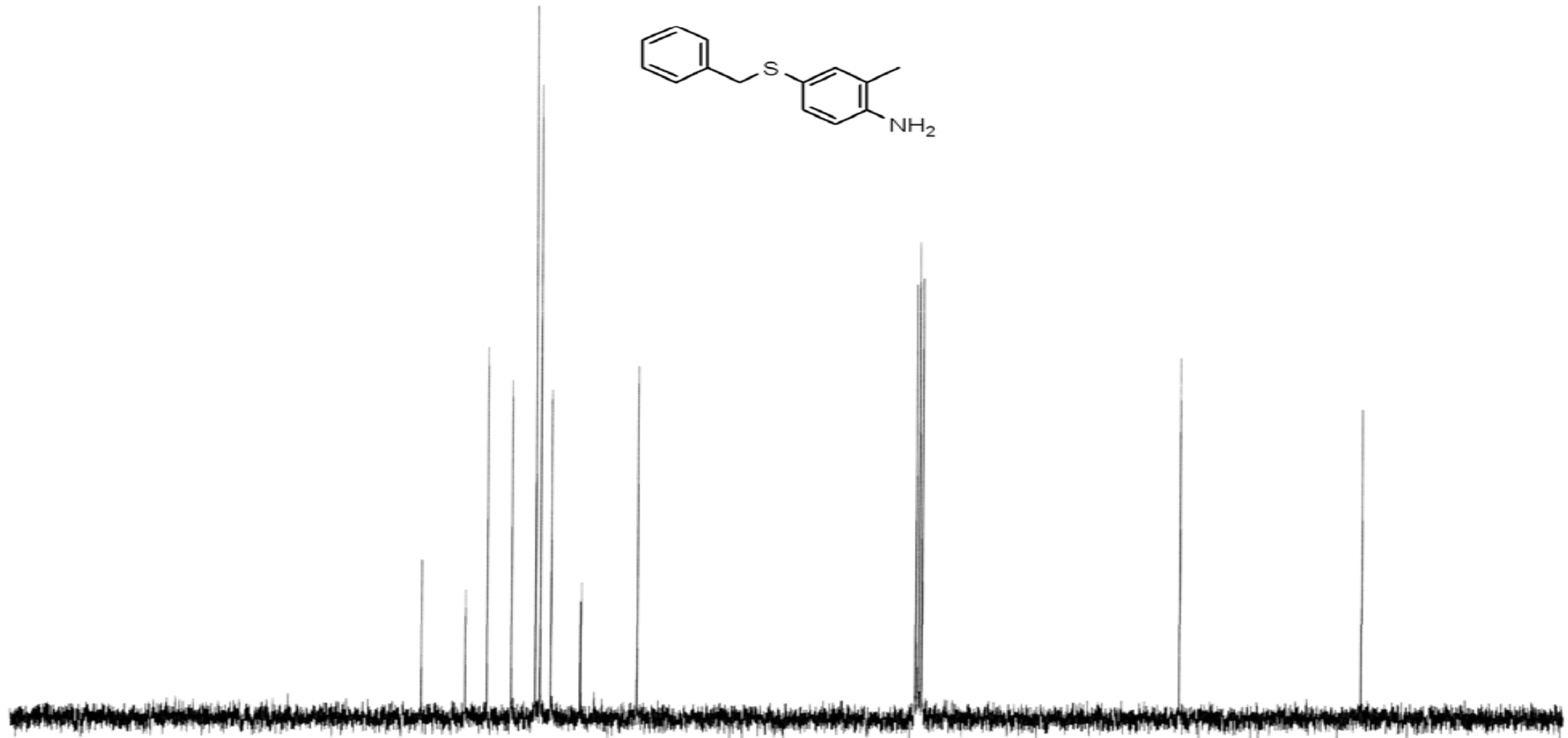


4-(4-Aminophenylsulfanyl)-benzonitrile (Table 2, entry 6)

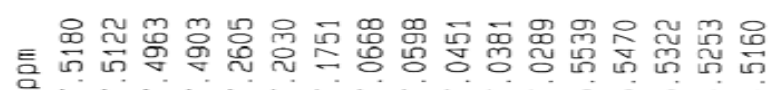

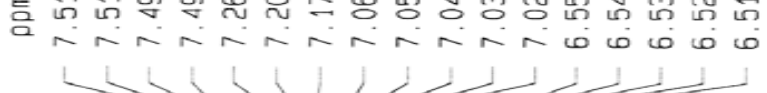

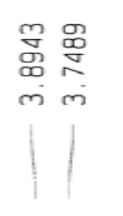

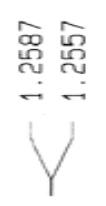

응

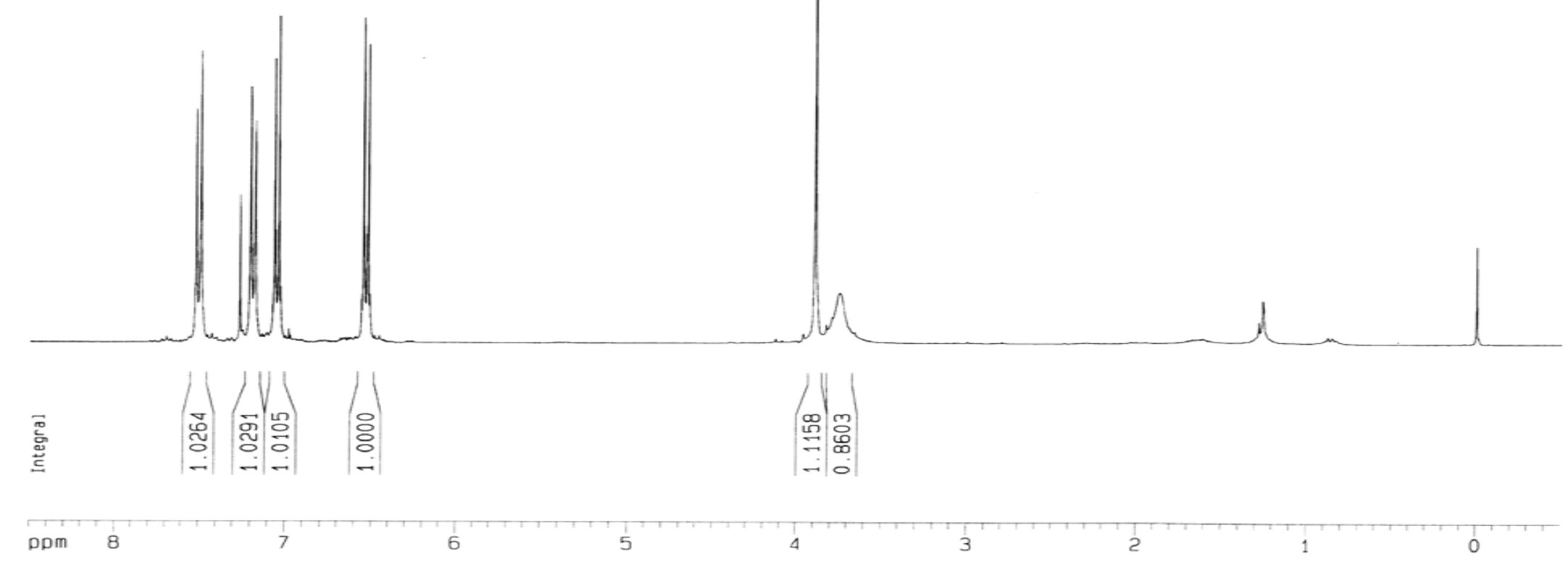

ppm 8

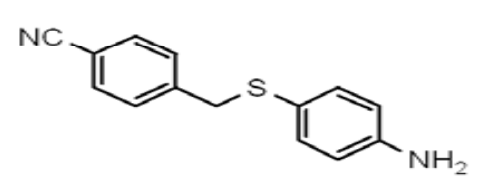

$\mathrm{NH}_{2}$

S21 


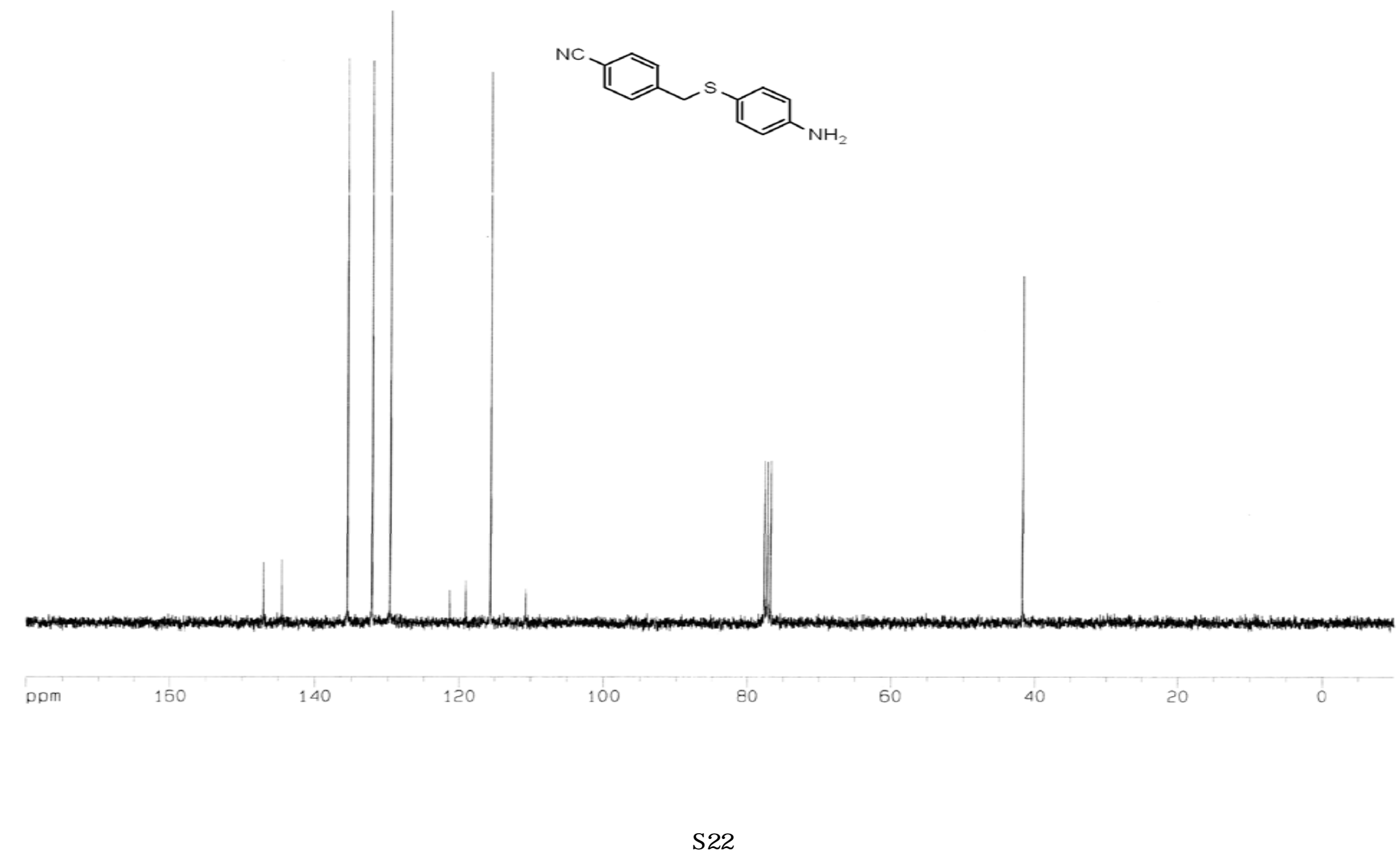


4-(2-Methyl-allylsulfanyl)-phenylamine (Table 2, entry 7)

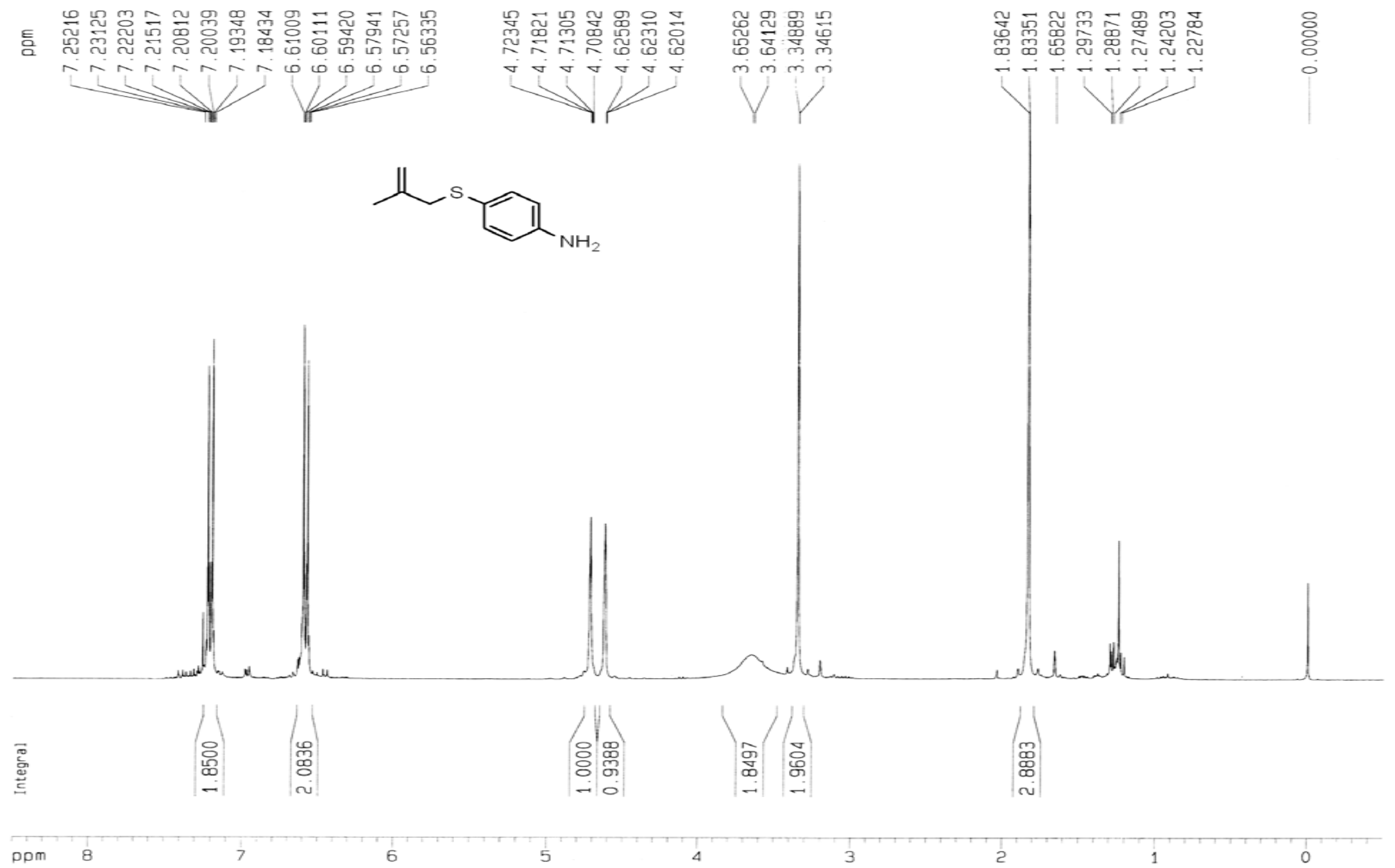




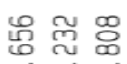

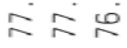

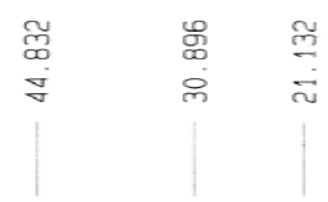

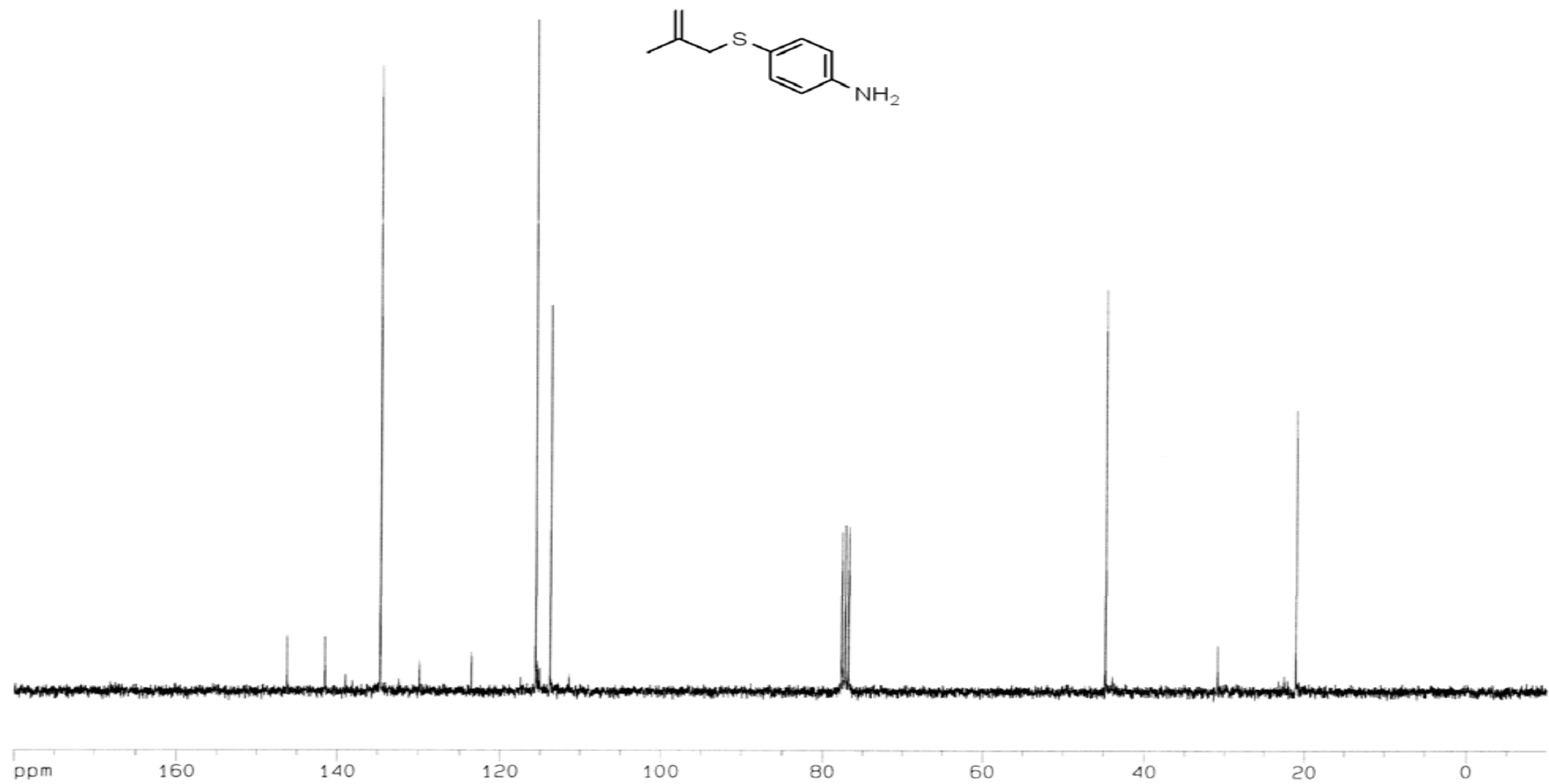


2-(4-Amino-phenylsulfanyl)-1-phenyl-ethanone (Table 2, entry 8, ref 4)
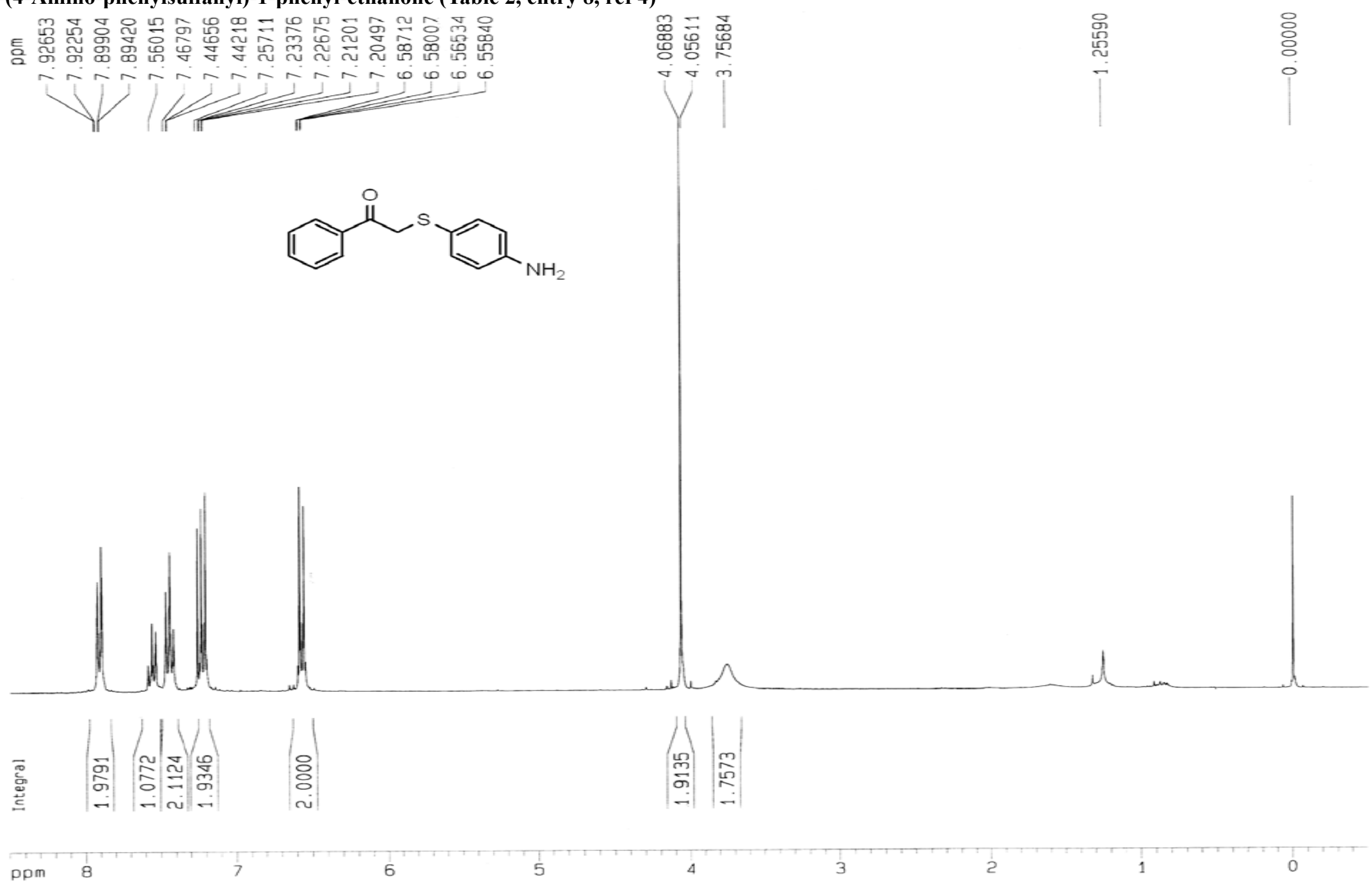

S25 
言变

ఫ ฌ

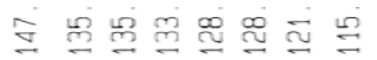

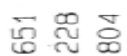

$\approx \approx ⿻ 上$

के
ก)

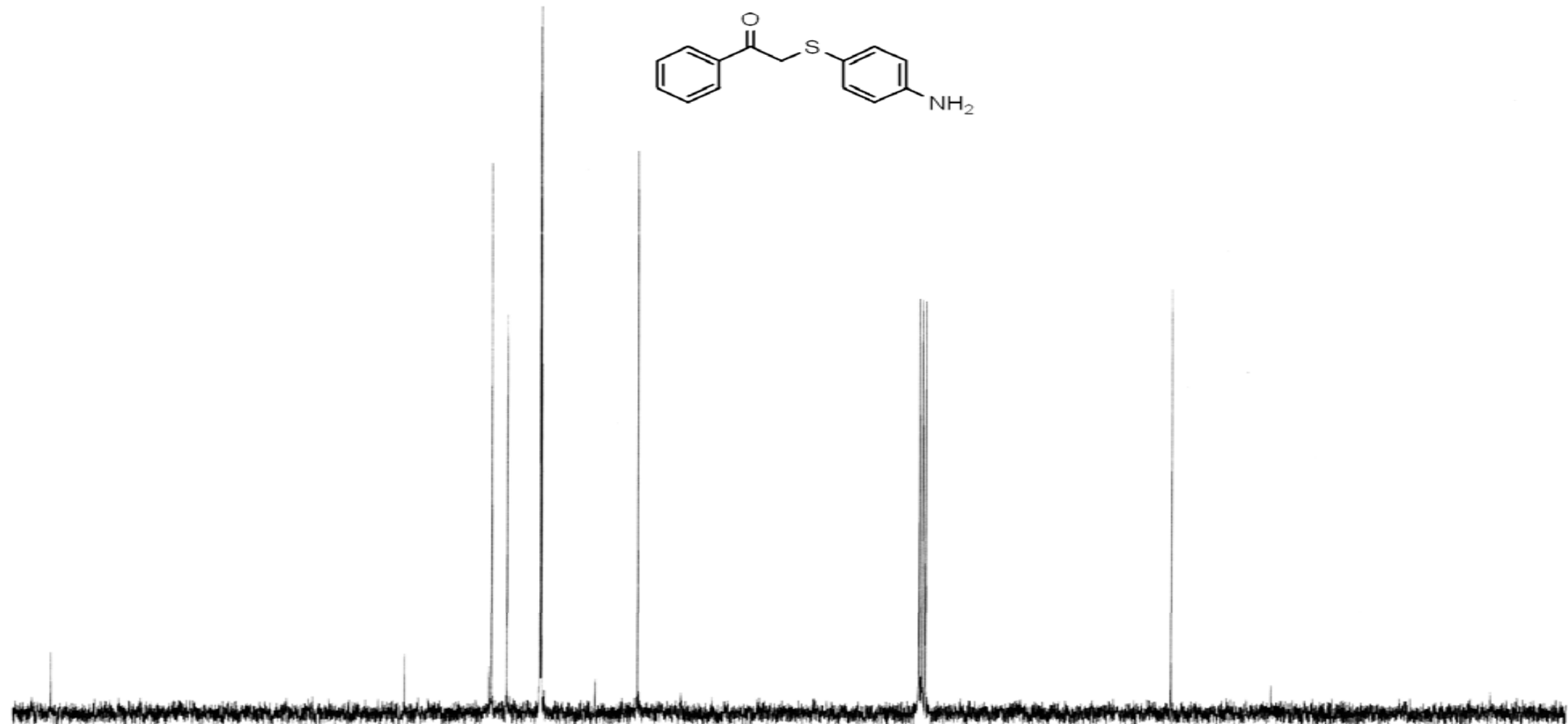

ppm

175

150

125

100

75

50 
(4-Amino-phenylsulfanyl)-acetic acid tert-butyl ester(Table 2, entry 9)

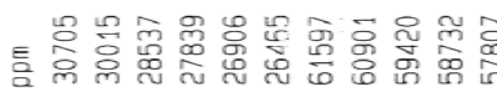

(1)
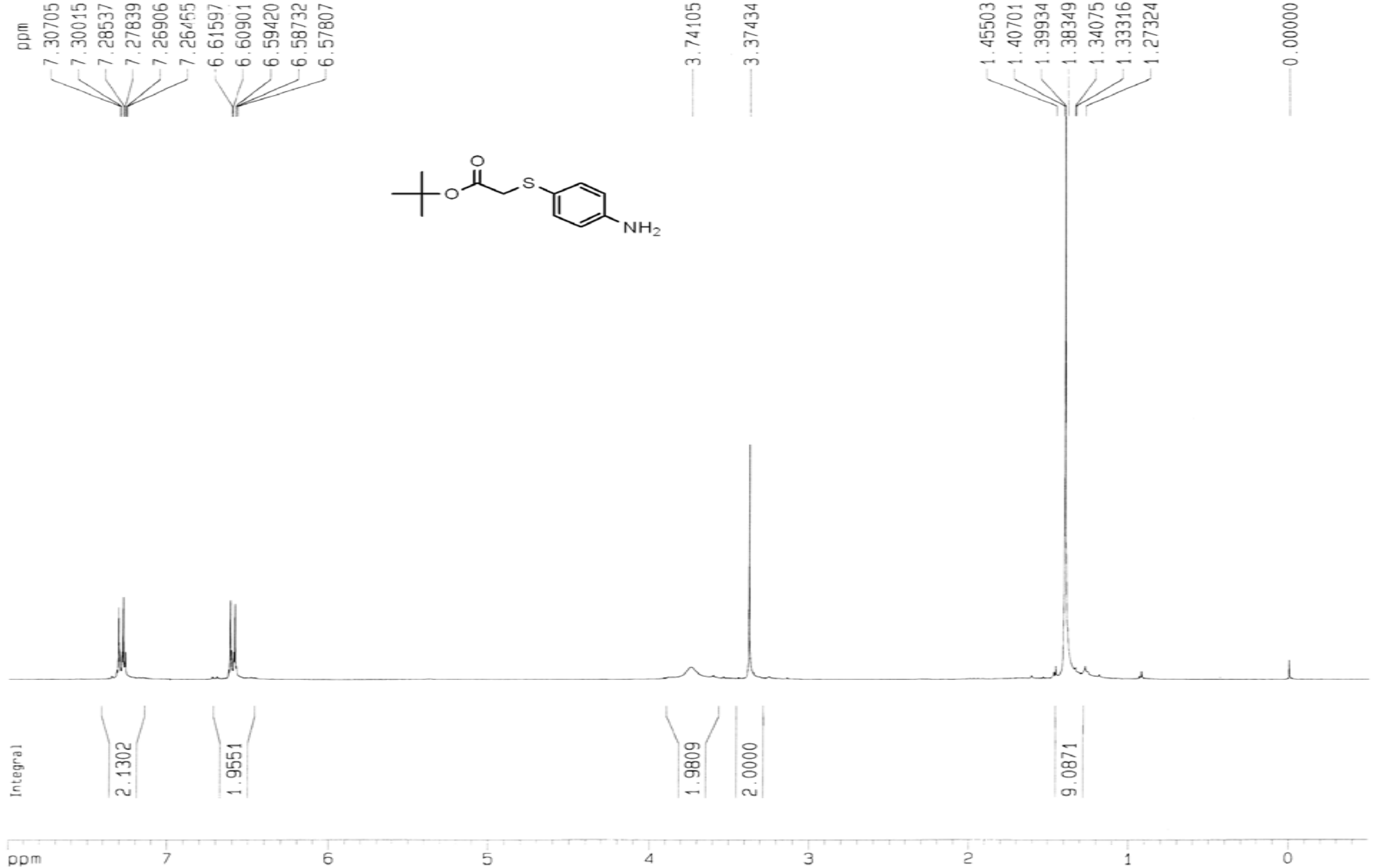


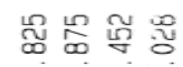

每

点学

十。美的

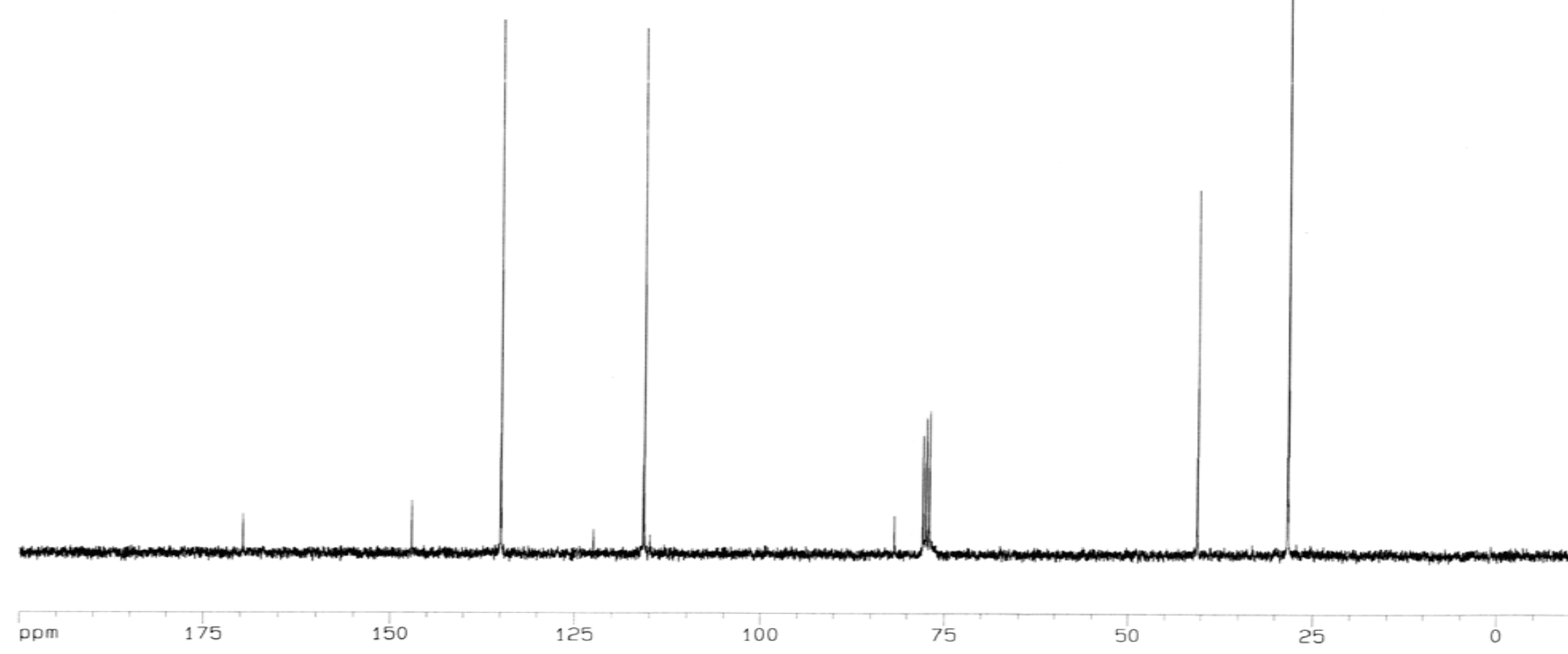


4-(5-Phenyl-pentylsulfanyl)-phenylamine (Table 2, entry 10)
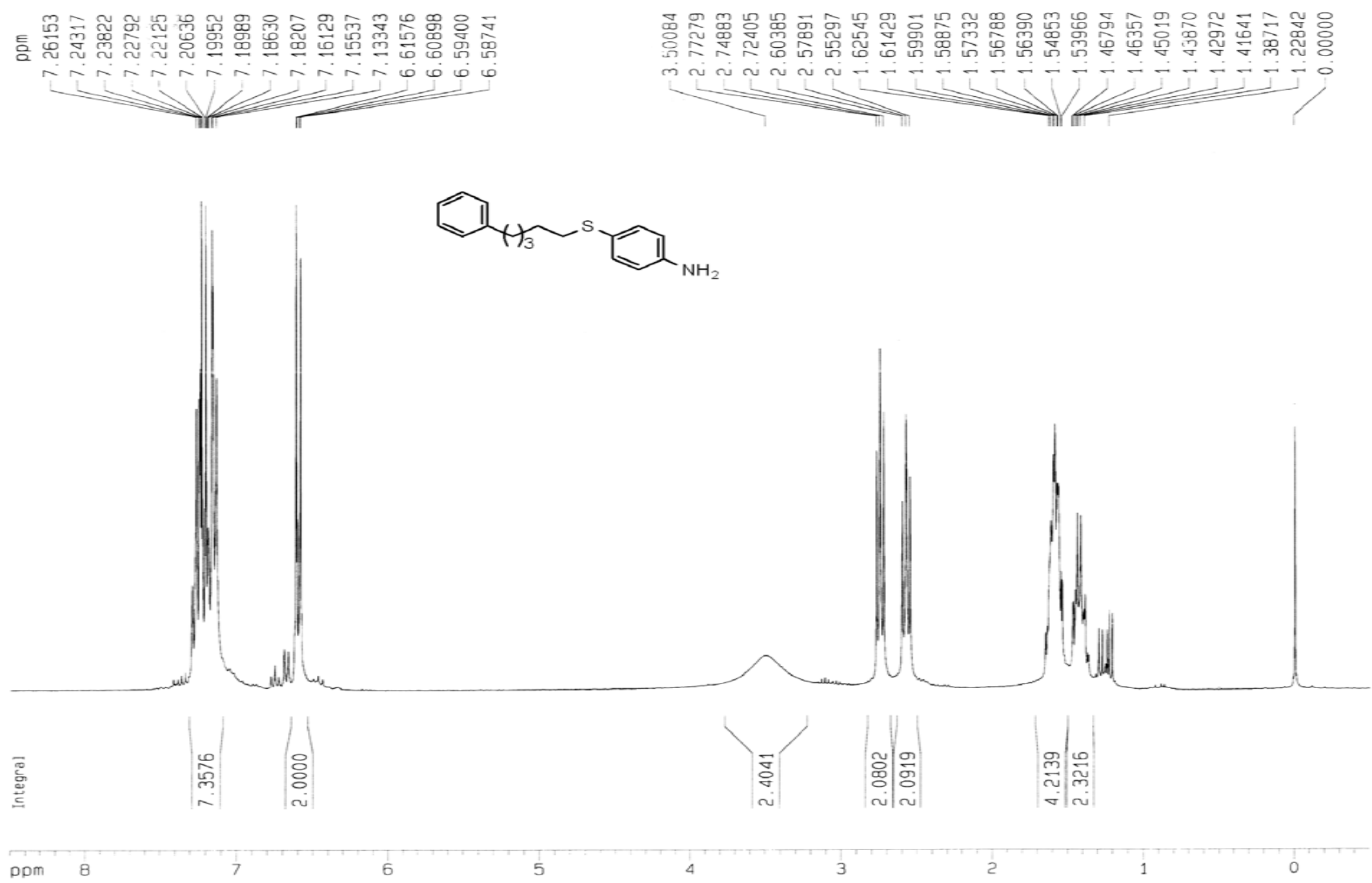

S29 
틈
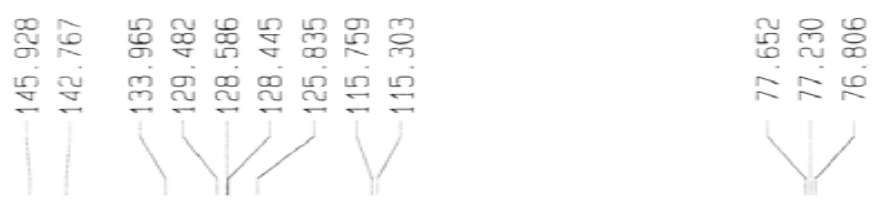

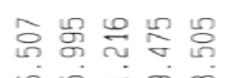

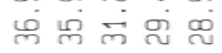

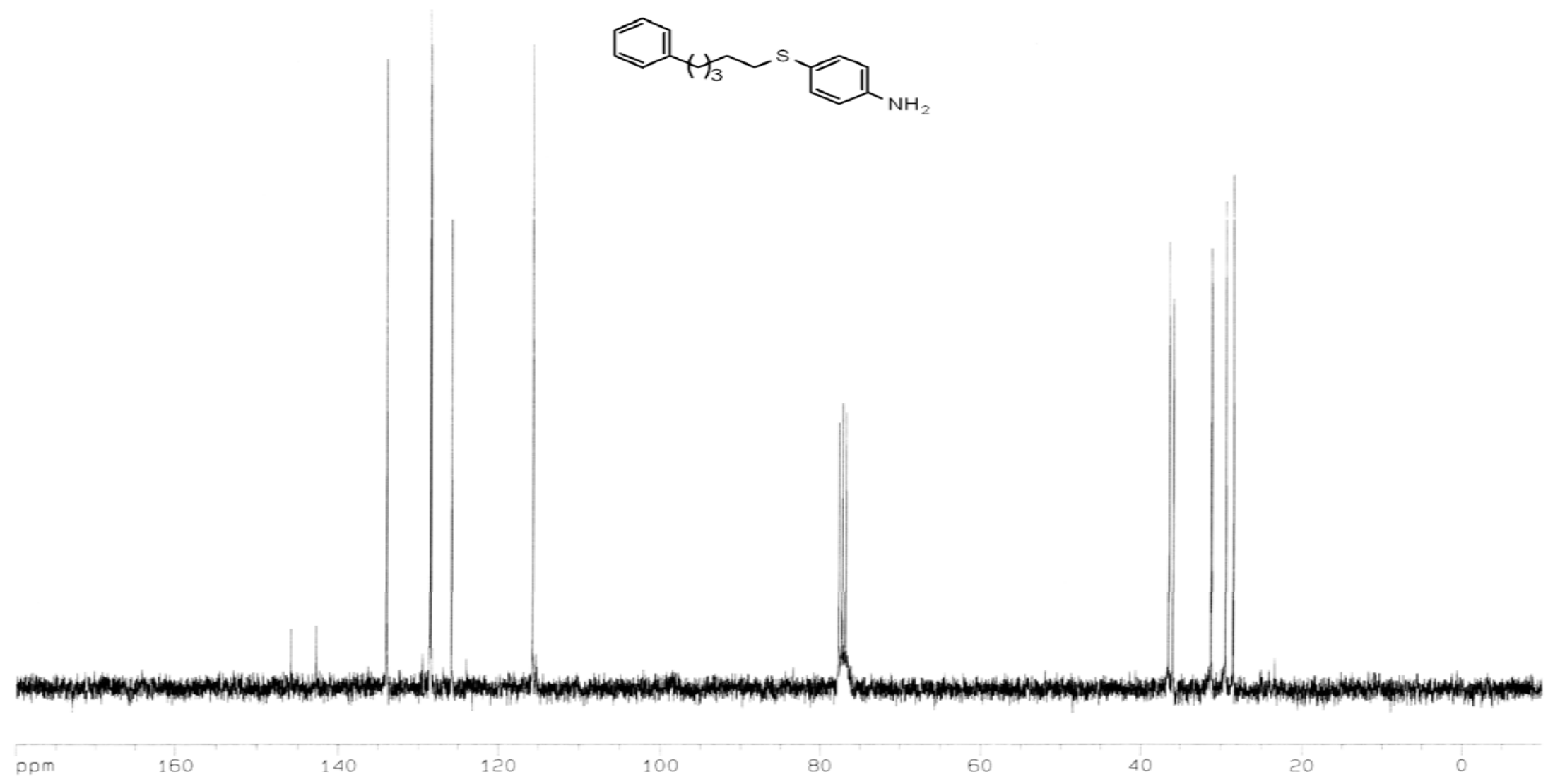

S30 


\section{4-Cyclohexylmethylsulfanyl-phenylamine (Table 2, entry 11)}
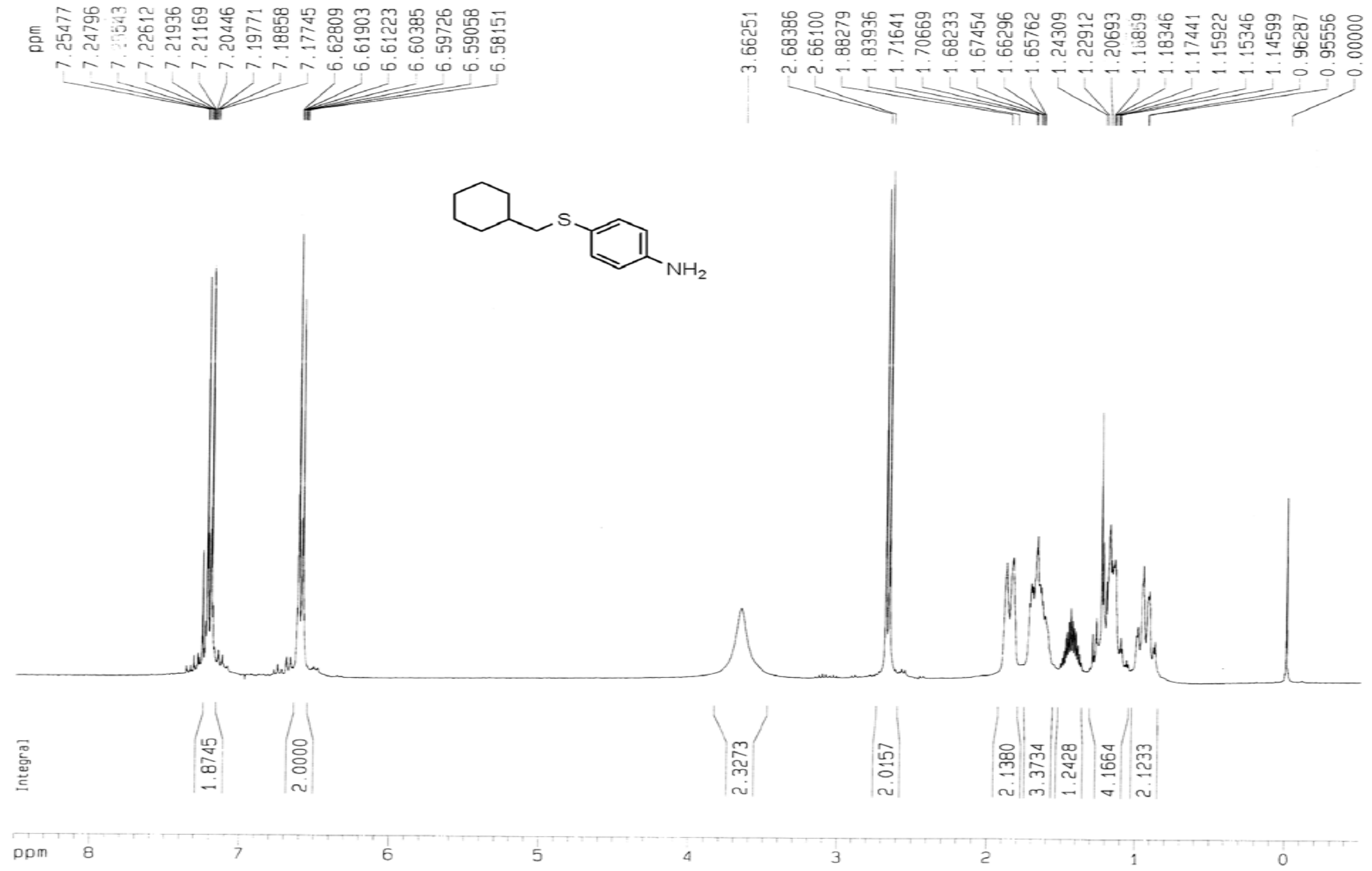
틈

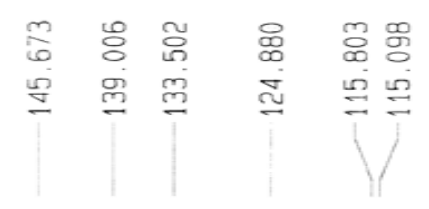

웅 ్ㅗㅇ

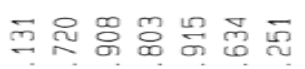

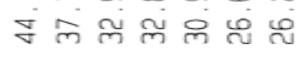
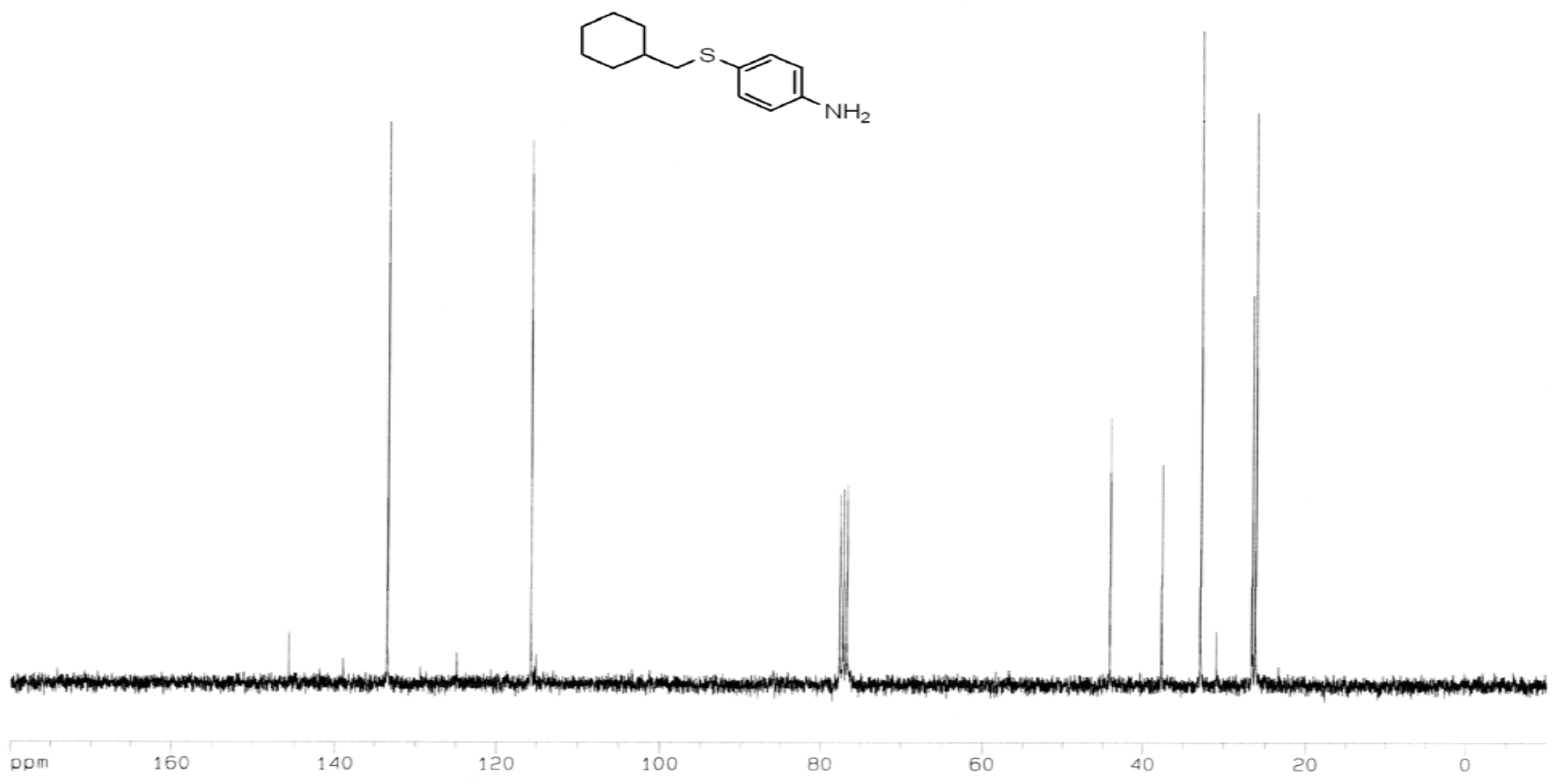

S32 
1-(4-Aminophenylsulfanyl)-hex-5-en-2-ol (Table 2, entry 12)

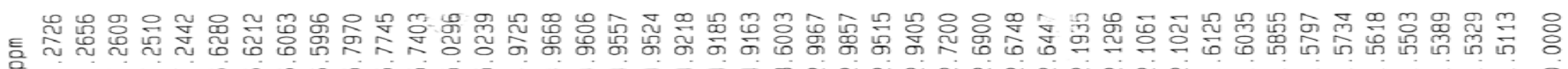

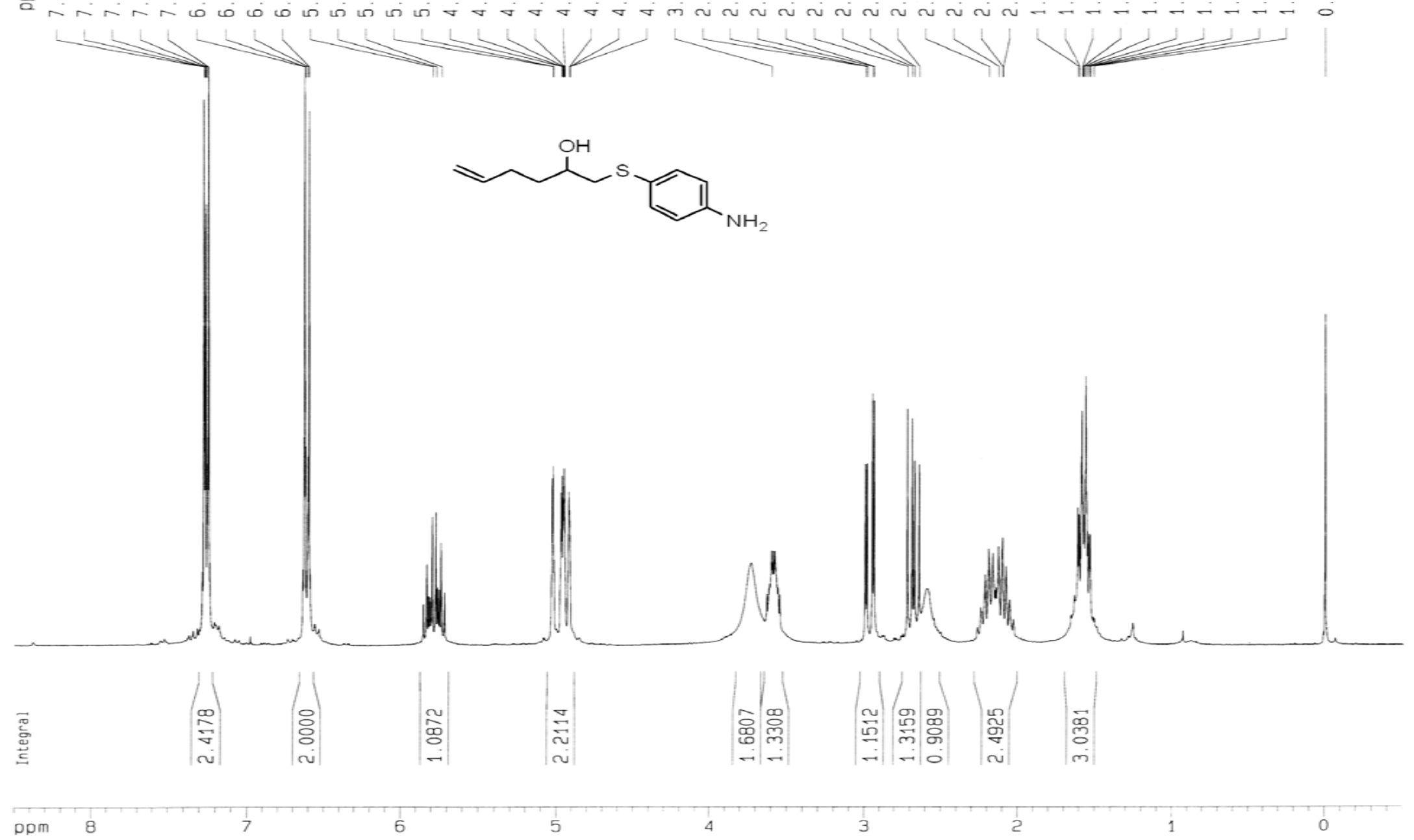

S33 


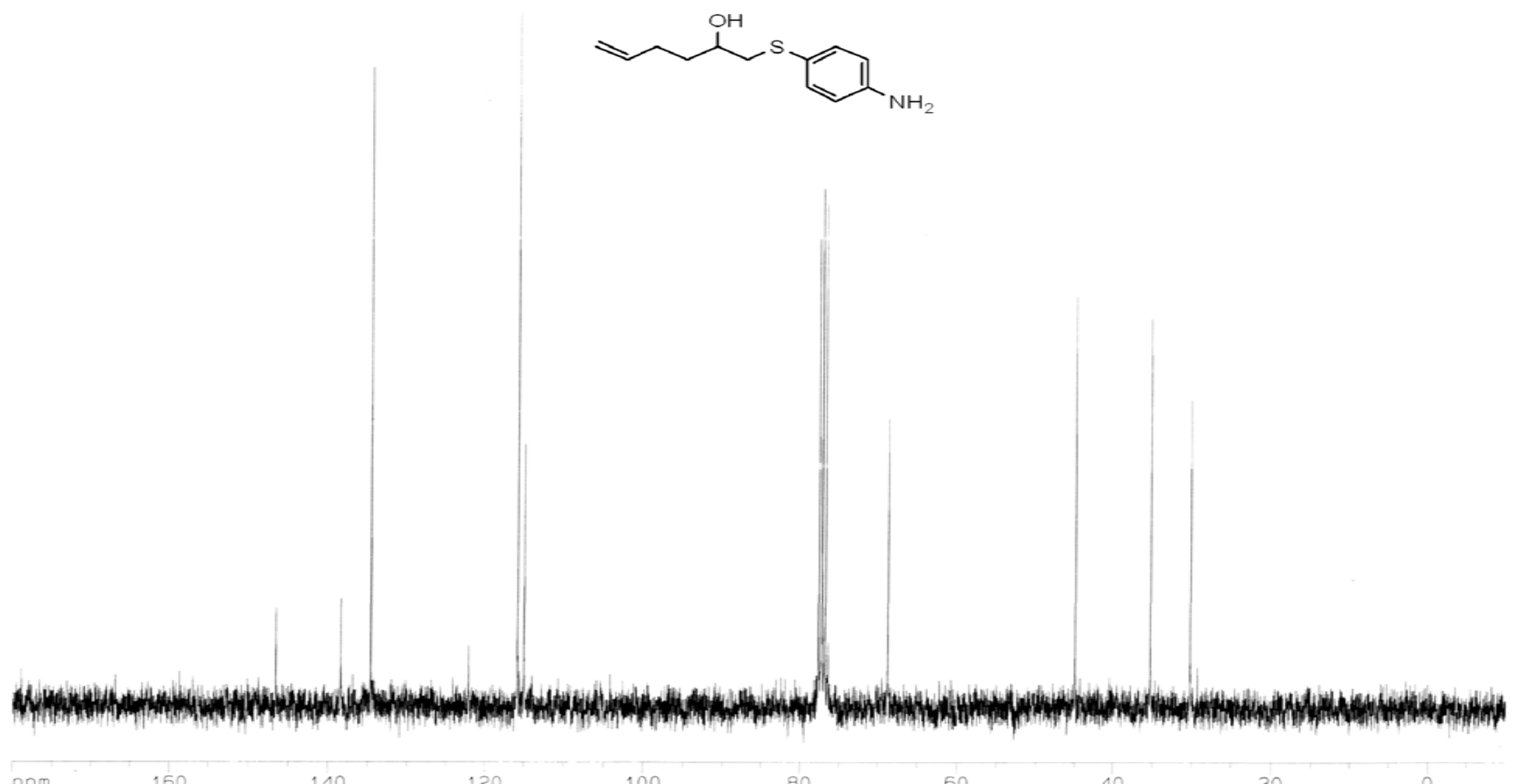


4-Benzylsulfanyl-benzylamine (Table 3, entry 1, ref 5)

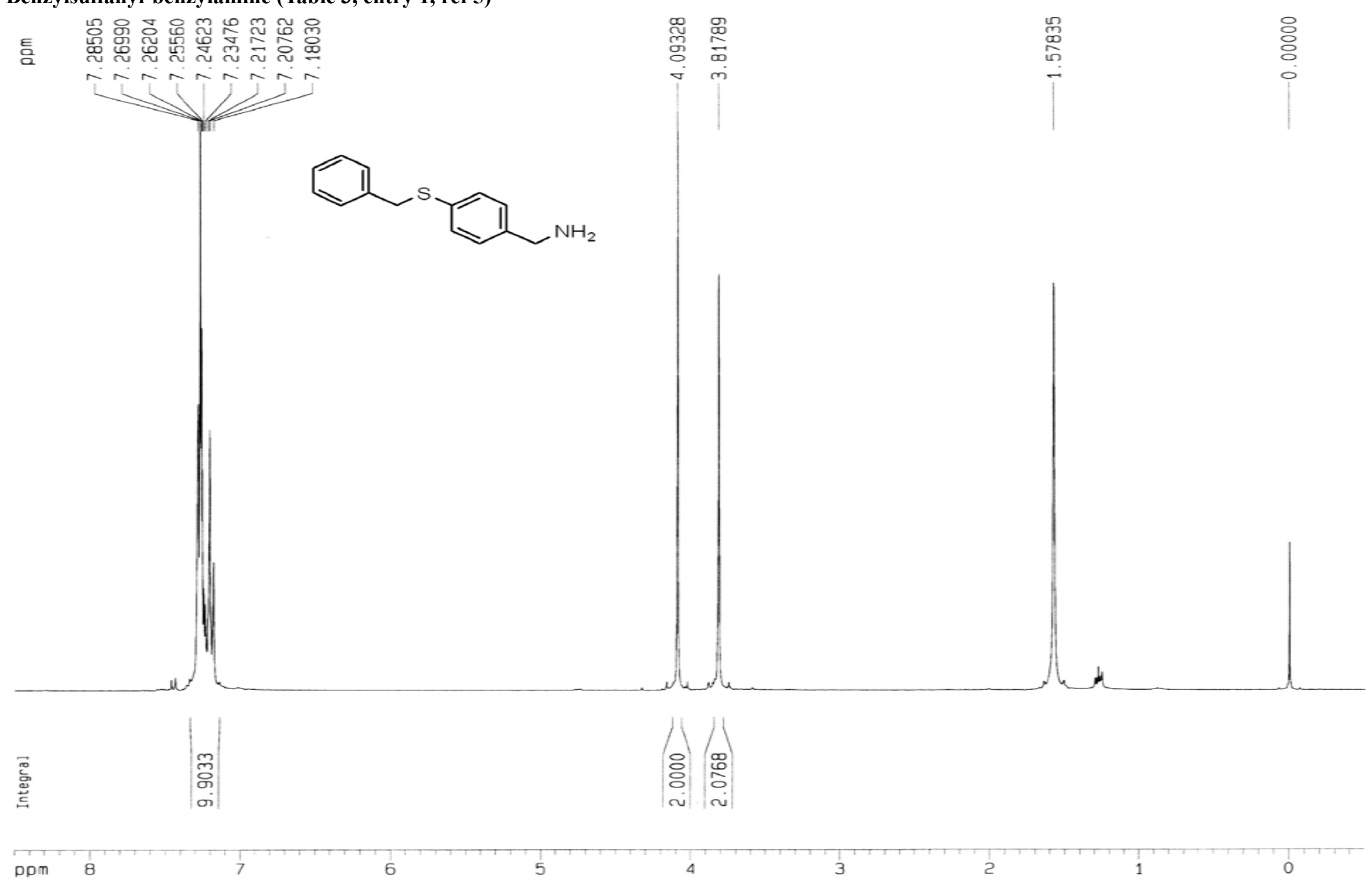




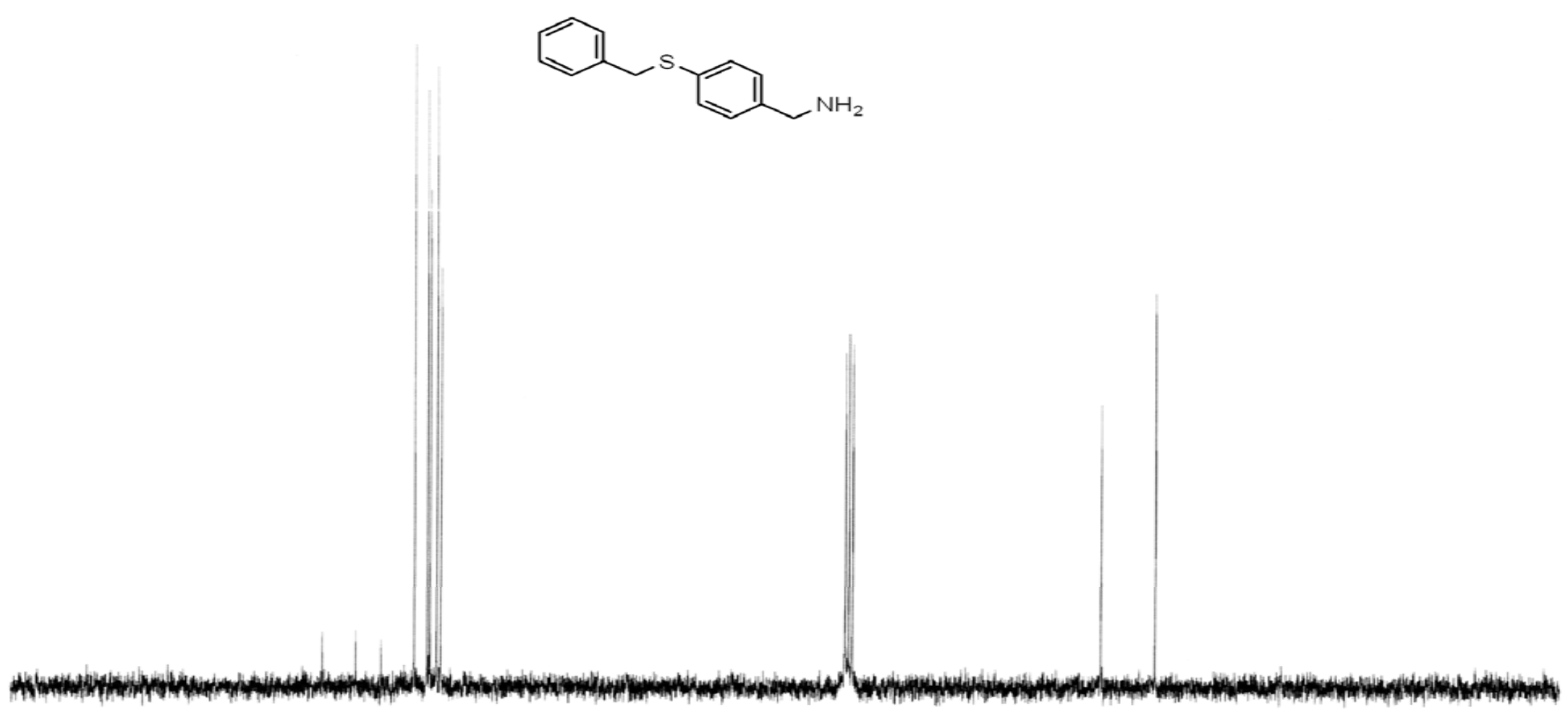
A How

140

120

100

80

60

40

20 
2-(4-Benzylsulfanyl-phenyl)-ethylamine (Table 3, entry 2)
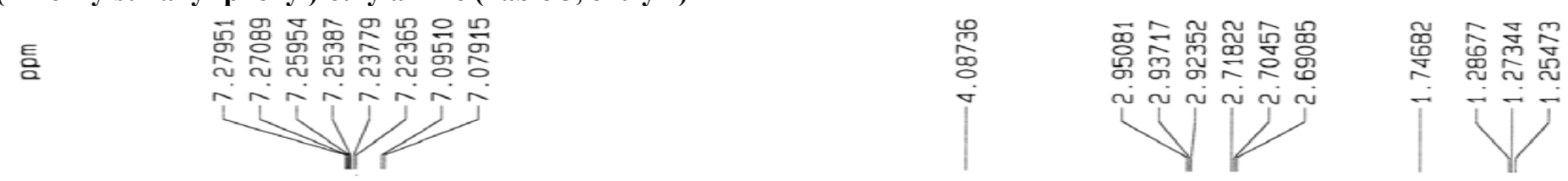

응

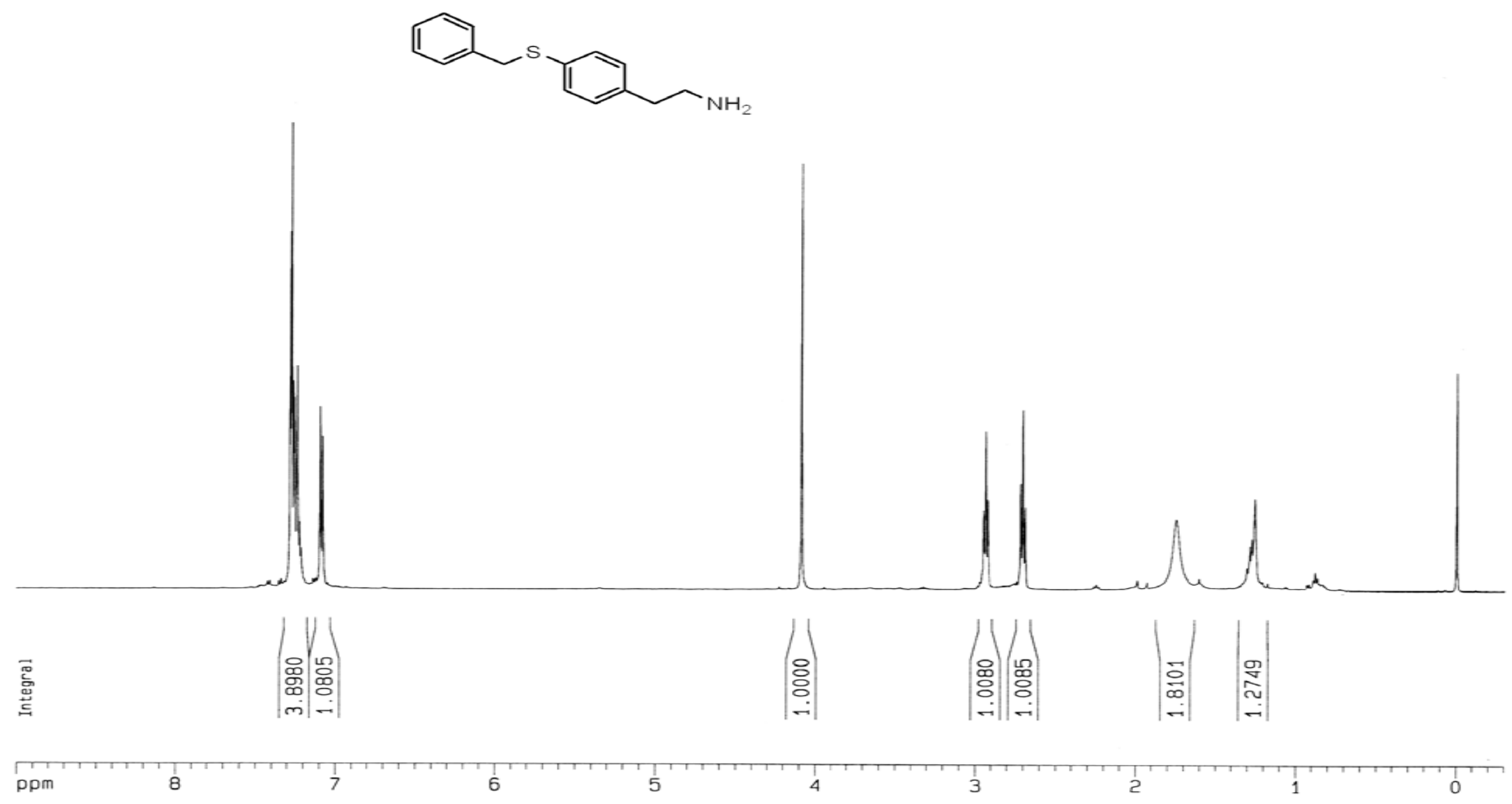


틈

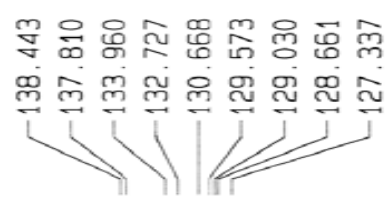
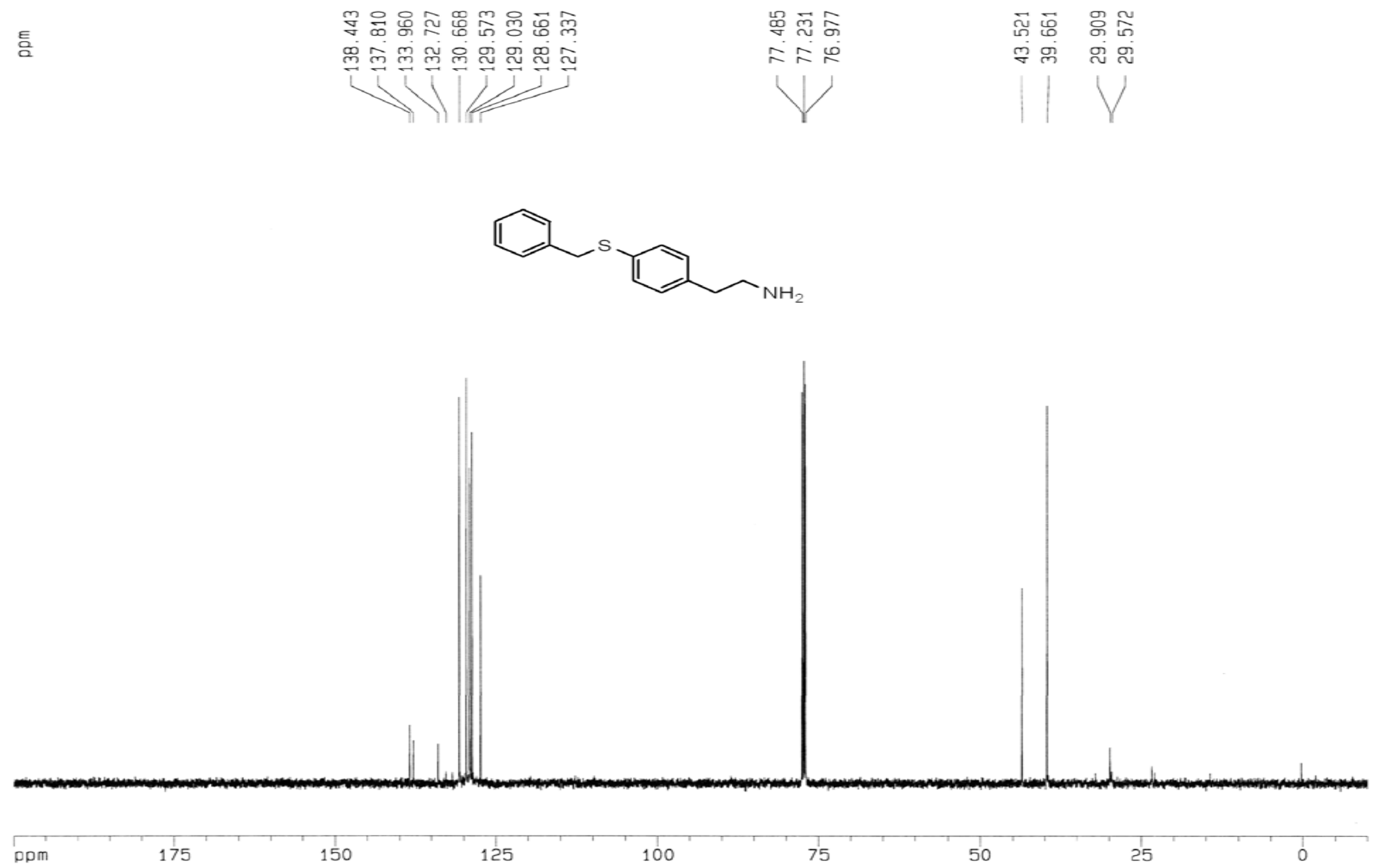

S38 
tert-Butyl [4-(2-aminoethyl)-phenylsulfany] acetate (Table 3, entry 3)

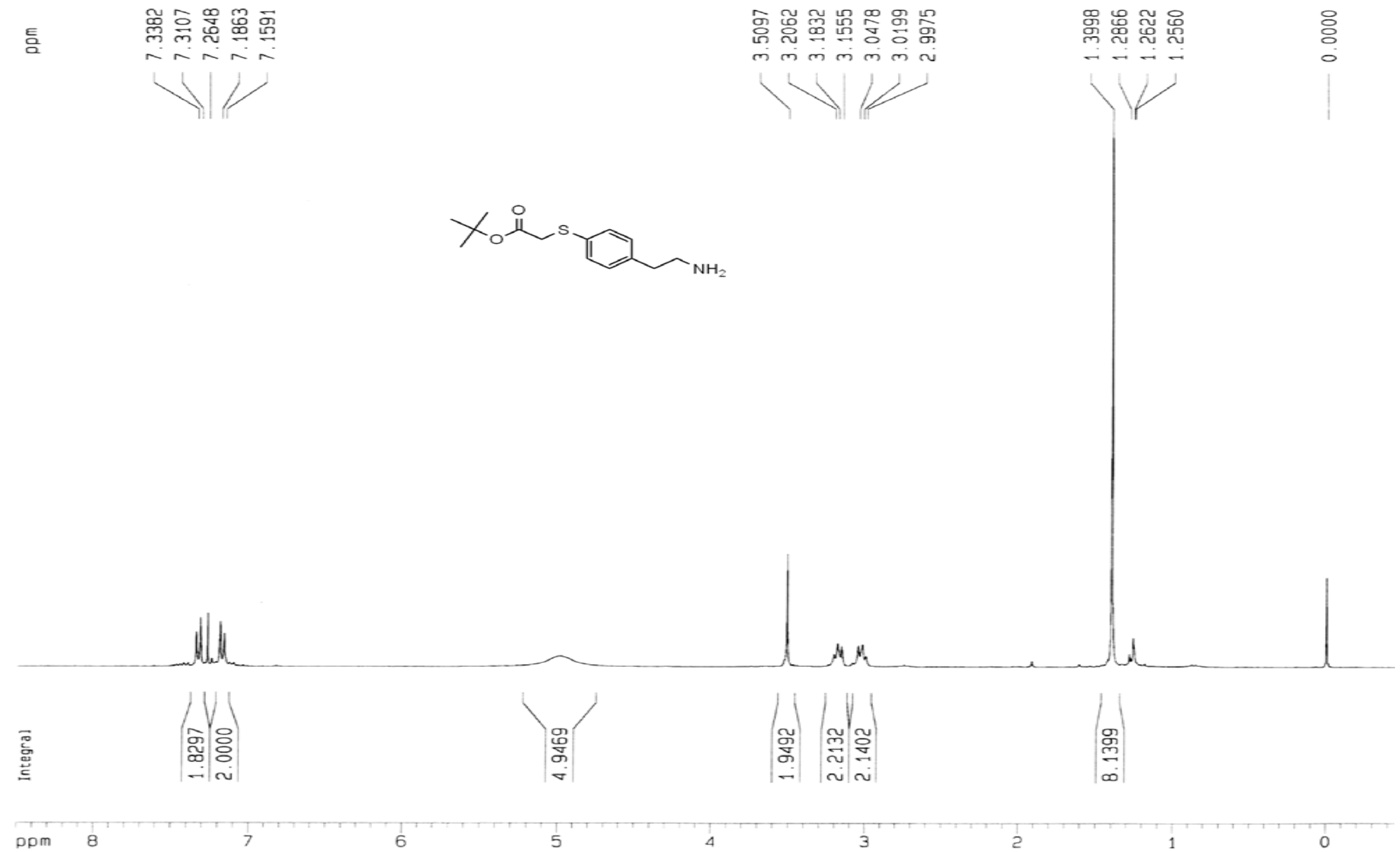



言 芩

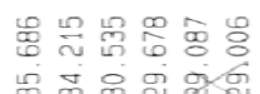
思要罚离

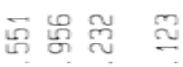
œ尺ミ心

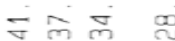

(

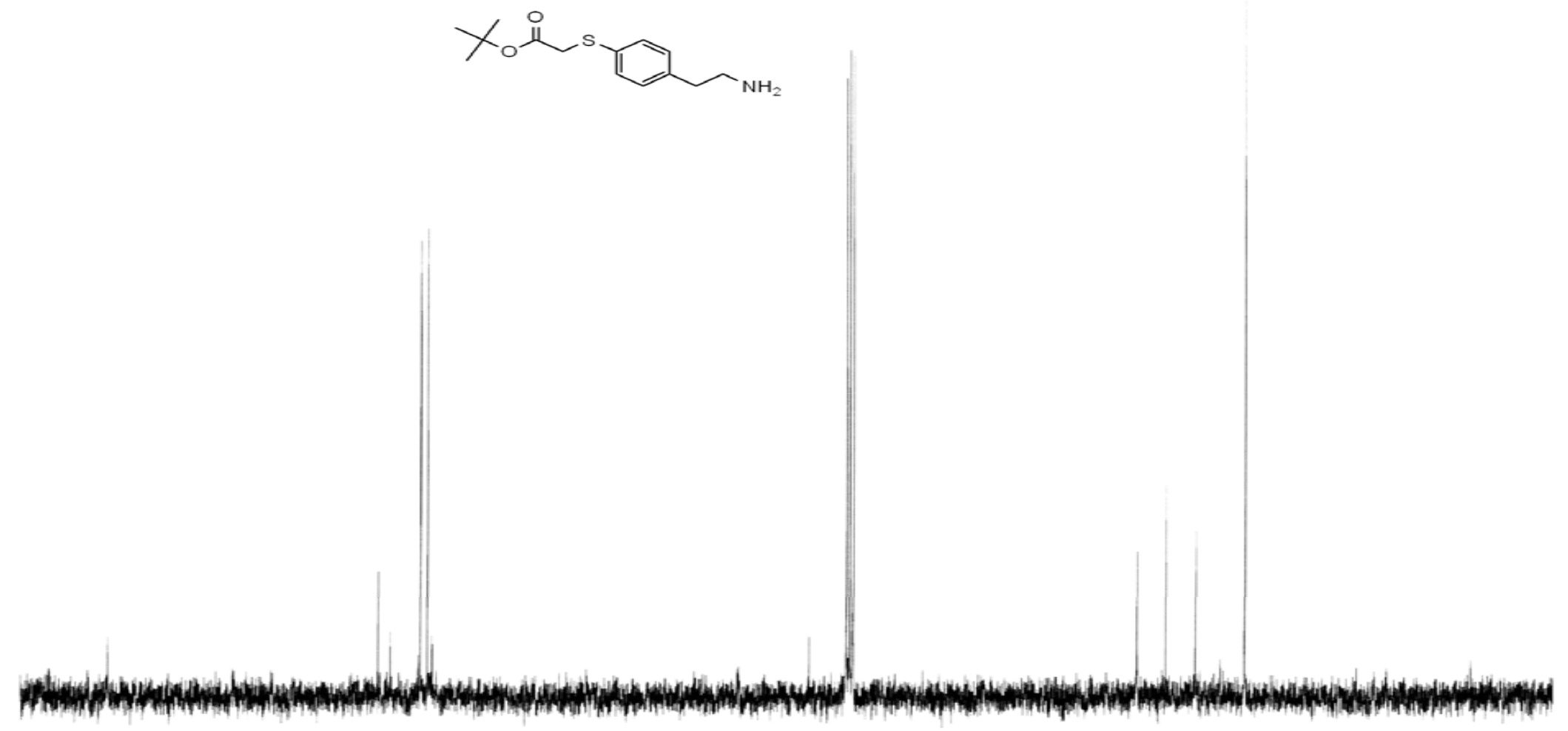

ppm

160

140

120

100

80

60

40

20 
tert-Butyl 2-[4-(2-aminoethyl)-phenylsulfanyl]-2-methylpropanoate (Table 3, entry 4, ref 6)

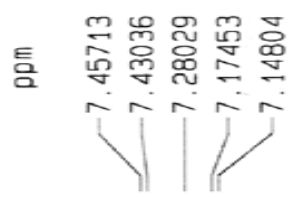
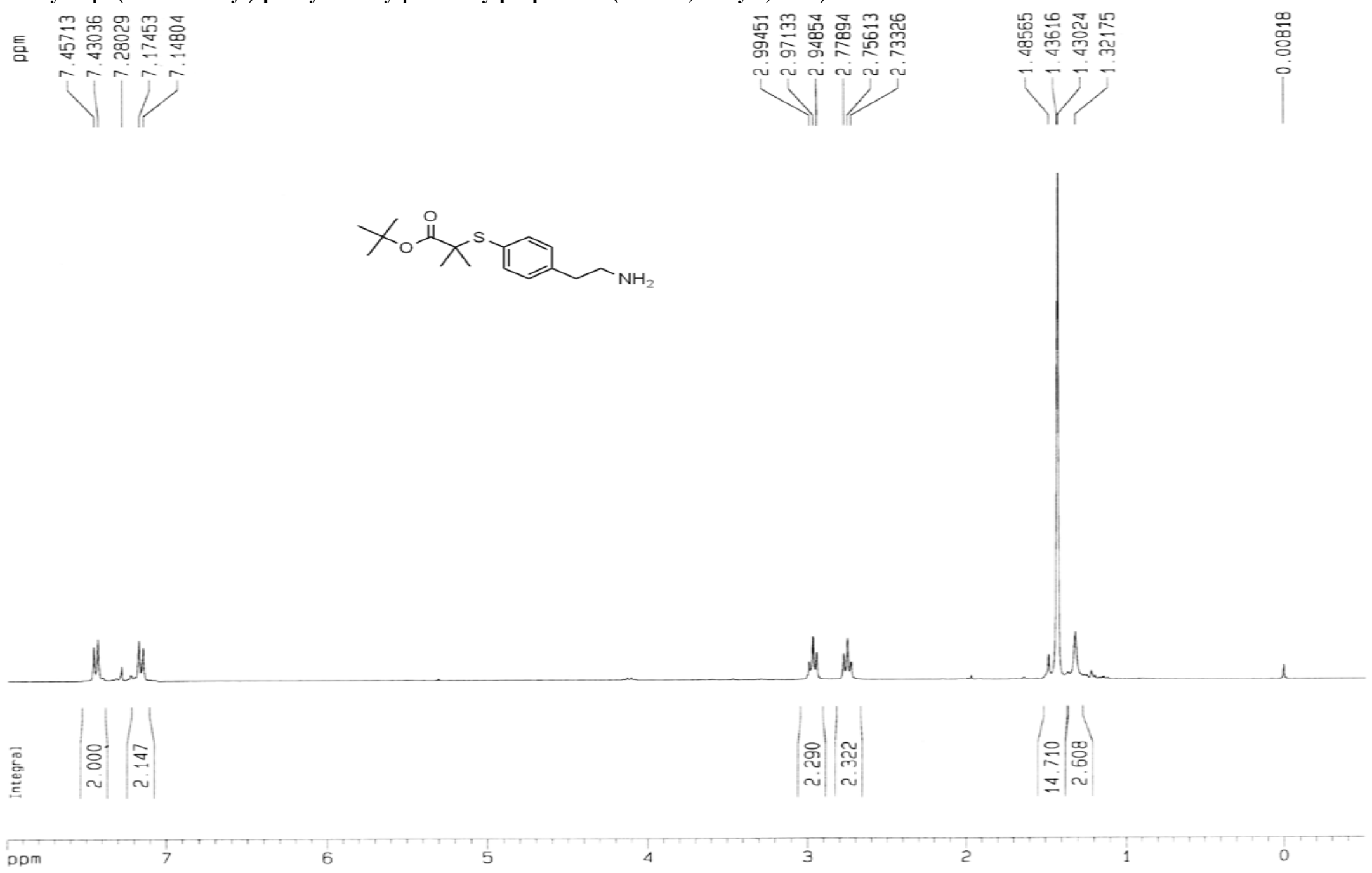

S41 


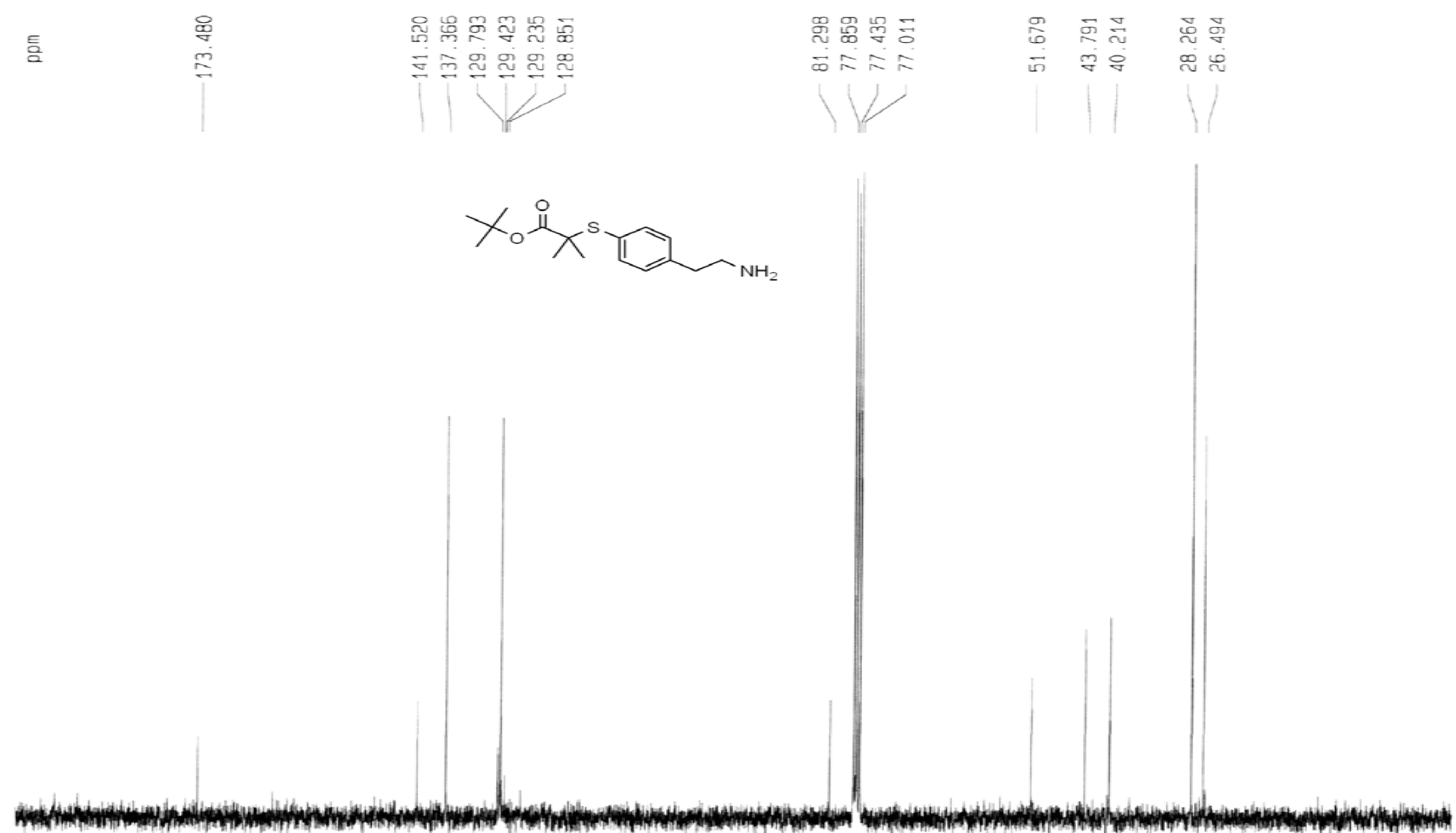

$175 \quad 150 \quad 125$

100

75

50

25 


\section{References}

1. Hucher, N.; Decroix, B.; Daich, A. J. Org. Chem. 2001, 66, 4695-4703.

2. Hansch, C.; Hathaway, B. A.; Gou, Z.; Selassie, C. D.; Dietrich, S. W.; Blaney, J. M.; Langridge, R.; Volz, K. W.; Kaufman, B. T. J. Med Chem. 1984, 27, 129-143.

3. Braunerova, G.; Buchta, V.; Silva, L.; Kunes, J.; Palat. Jr., K. IL FARMACO, 2004, 59, 443-450.

4. Waldron, W. R.; Reid, E. H. J. Am. Chem. Soc. 1923, 45, 2399-2417.

5. Euerby, M. R.; Waigh, R. D. Journal of Chemical Research (Synopses), 1982, 9, 240-241.

6. Brown, P. J.; Winegar, D. A.; Plunket, K. D.; Moore, L. B.; Lewis, M. C.; Wilson, J. G.; Sundseth, S. S.; Koble, C. S.; Wu, Z.; Chapman, J. M.; Lehmann, J. M.; Kliewer, S. A.; Wilson, T. M. J. Med. Chem. 1999, 42, 3785-3788. 